\title{
Rényi entanglement entropy of Fermi and non-Fermi liquids: Sachdev-Ye-Kitaev model and dynamical mean field theories
}

\author{
Arijit Haldar $\odot,{ }^{1,2, *}$ Surajit Bera $\odot,{ }^{1}$ and Sumilan Banerjee $\odot^{1, \dagger}$ \\ ${ }^{1}$ Centre for Condensed Matter Theory, Department of Physics, Indian Institute of Science, Bangalore 560012, India \\ ${ }^{2}$ Department of Physics, University of Toronto, 60 St. George Street, Toronto, Ontario, M5S 1A7, Canada
}

(Received 1 May 2020; accepted 19 August 2020; published 28 September 2020)

\begin{abstract}
We present a method for calculating Rényi entanglement entropies for fermionic field theories originating from microscopic Hamiltonians. The method builds on an operator identity, which leads to the representation of traces of operator products, and thus Rényi entropies of a subsystem, in terms of fermionic-displacement operators. This allows for a very transparent path-integral formulation, both in and out of equilibrium, having a simple boundary condition on the fermionic fields. The method is validated by reproducing well-known expressions for entanglement entropy in terms of the correlation matrix for noninteracting fermions. We demonstrate the effectiveness of the method by explicitly formulating the field theory for Rényi entropy in a few zero- and higher dimensional large- $N$ interacting models akin to the Sachdev-Ye-Kitaev (SYK) model and for the Hubbard model within the dynamical mean field theory (DMFT) approximation. We use the formulation to compute Rényi entanglement entropy of interacting Fermi liquid (FL) and non-Fermi liquid (NFL) states in the large- $N$ models and compare the results successfully with those obtained via exact diagonalization for finite $N$. We elucidate the connection between Rényi entanglement entropy and residual entropy of the NFL ground state in the SYK model and extract sharp signatures of quantum phase transition in the entanglement entropy across an NFL to FL transition. Furthermore, we employ the method to obtain nontrivial system-size scaling of entanglement in an interacting diffusive metal described by a chain of SYK dots.
\end{abstract}

DOI: 10.1103/PhysRevResearch.2.033505

\section{INTRODUCTION}

Quantum entanglement has emerged as a major tool to characterize quantum phases and phase transitions [1-6] and to distill the fundamental quantum mechanical nature of nontrivial many-body states, e.g., those with topological order that is otherwise hard to quantify $[7,8]$. Recent developments in condensed-matter and high-energy physics have revealed beautiful connections among entanglement, thermalization, and dynamics, leading to classification of dynamical phases of interacting quantum systems into thermal and many-body localized (MBL) phases [9-11]. Quantum entanglement is quantified in terms of properties of the reduced density matrix, $\rho_{A}=\operatorname{Tr}_{B} \rho$, of a system with density matrix $\rho$ and divided, e.g., into subsystems $A$ and $B$, where $\operatorname{Tr}_{B}$ is the partial trace over the degrees of freedom of $B$. Typical entanglement measures constructed out of $\rho_{A}$ for a pure state are von Neumann and Rényi entanglement entropies. The latter can be used to compute useful measures [12-16] for entanglement even in a thermal mixed state.

\footnotetext{
*arijit.haldar@utoronto.ca

†sumilan@iisc.ac.in

Published by the American Physical Society under the terms of the Creative Commons Attribution 4.0 International license. Further distribution of this work must maintain attribution to the author(s) and the published article's title, journal citation, and DOI.
}

However, calculation of entanglement entropy is much more challenging than, e.g., that of thermal entropy or usual correlation functions. Over the past decade, a lot of progress has been made to obtain entanglement entropy, both numerically and analytically, for noninteracting bosonic and fermionic systems $[3,4,17]$, and at critical points described by conformal field theories $[1,2]$. The latter rely on field-theoretic techniques using replicas and path integrals, typically in imaginary time, with complicated boundary conditions on fields and associated Green's function along the time direction [1-3]. Such methods are often hard to implement for interacting systems. Hence, computation of entanglement entropy for interacting systems are only limited to small systems using exact diagonalization (ED) or for systems like those without sign problem accessible via quantum Monte Carlo (QMC) simulations [18-21].

A promising path-integral approach that circumvents the use of complicated boundary conditions using the known relation between reduced density matrix and Wigner characteristic function [22] has been recently proposed for bosonic systems in Ref. [23]. Motivated by this, here we develop a field-theoretic method to compute Rényi entanglement entropy for fermions. A similar field-theoetic formalism for fermions has been developed independently by Moitra and Sensarma [24]. To this end, we derive different representations of an operator and traces of product of operators in terms of fermionic displacement operators [25]. The representations allow us to develop a very transparent fermionic coherent-state path-integral method with simple boundary 
condition to compute Rényi entropies of a subregion of the system in terms of a fermionic version of the Wigner characteristic function. The formalism can equally be applied to calculate subsystem Rényi entropy for thermal equilibrium state via imaginary-time path integral or nonequilibrium time evolution described via Schwinger-Keldysh field theory. The approach naturally transcends the effect of boundary condition into time-dependent self-energy, which acts like a kick at a particular time. We show that the method immediately reproduces the known expressions for von Neumann and Rényi entanglement entropies for noninteracting fermions. The effect of the time-dependent self-energy can be implemented for interacting systems treated within standard perturbative and nonperturbative field-theoretic approximation and diagrammatic continuous-time Monte Carlo simulation [26]. We elucidate this by deriving the subsystem second Rényi entropy within two well-known approaches to treat correlated fermions: (a) strongly interacting large- $N$ fermionic models based on Sachdev-Ye-Kitaev model [27,28] and (b) dynamical mean-field theory (DMFT) [29].

In the other major part of the paper, we explicitly demonstrate the utility of the method by computing the second Rényi entropy $\left(S^{(2)}\right)$ for subsystems in several large- $N$ model in thermal equilibrium: (i) zero-dimensional SYK model having infinite-range random four-fermion or two-body interaction with a non-Fermi liquid (NFL) ground state, (ii) SYK model with additional quadratic hopping between fermions having a Fermi liquid (FL) ground state, (iii) a generalized SYK model, the Banerjee-Altman (BA) model [30], having quantum phase transition (QPT) between SYK NFL and FL, and (iv) an extended system, a chain of SYK dots [31,32], describing an interacting diffusive metal. We compute the subsystem Rényi entropy at the large- $N$ saddle point as a function of subsystem size for the thermal density matrix at a temperature $T$ and extrapolate to $T \rightarrow 0$ to obtain ground-state Rényi entanglement entropy. In all the above cases except (iii), we compare and contrast the results for the interacting systems with a corresponding noninteracting system where the SYK interaction is replaced by infinite-range random hopping. For the noninteracting models, we numerically calculate the second Rényi entropy by numerical diagonalization for moderately large systems and compare with large- $N$ field theoretic results. Moreover, we also compute subsystem Rényi entropy via exact diagonalization (ED) of many-body Hamiltonian for small $N \approx 8-16$ for the interacting zero-dimensional models. We obtain the following important results using our method for the large- $N$ models:

(1) We show that for the SYK model, in the $N \rightarrow \infty$ limit, the zero-temperature residual entropy [27,33-35] of the SYK NFL contributes to the $T=0$ subsystem Rényi entropy, thus making it difficult to recover the true quantum entanglement of the NFL ground state starting from a thermal ensemble. Moreover, consistent with our numerical results, we analytically prove that the SYK model is maximally entangled when the relative size of the subsystem $p \rightarrow 0$.

(2) We demonstrate how the $T \rightarrow 0$ bipartite Rényi entanglement entropy crosses over from a NFL to a FL as function of the strength of hopping in the SYK model with random quadratic term. The results show that heavy FL are much more entangled than weakly or noninteracting FL.
(3) In the BA model with a NFL-FL transition, we establish a precise connection between subsystem Rényi entropy and the residual entropy of the NFL and show that the Rényi entropy also carries sharp signature of the underlying QPT, like the residual entropy [30].

(4) In the extended one-dimensional (1D) model of SYK dots, we compute Rényi entanglement entropy of a subsystem of length $l$. We find a crossover from $S^{(2)} \sim \ln l$ to $S^{(2)} \sim$ $\ln \left[1 /\left(l^{-2}+l_{0}^{-2}\right)^{1 / 2}\right]$ with increasing $l$ where the $\ln l$ behavior, expected for gapless fermions, gets cut off by an emergent mean-free path $l_{0}$ in an interacting diffusive metal.

The paper is organized as follows. In Sec. II A, we derive the operator identities that form the basis of our formalism and discuss the connections of these identities with Rényi entropy of a subsystem. The general formulation for the equilibrium and nonequilibrium path integrals to compute the Rényi entropy is discussed in Sec. II B. Section IIC describes the application of the field-theory formalism to derive well-known formulas for Rényi entropy of noninteracting fermions. In Secs. II D and II E, we develop the field theory for Rényi entropy in several interacting large- $N$ models based on the SYK model and in the Hubbard model within DMFT approximation, respectively. We describe our analytical and numerical results for Rényi entropy in the large- $N$ models and compare the large- $N$ results with those obtained from numerical exact diagonalization in Sec. III. Additional details of the derivations of the operator identities, path-integral formulations, and their analytical and numerical implementations in various models for computing Rényi entropies are given in the Appendixes.

\section{COHERENT STATE-PATH INTEGRAL FORMALISM FOR FERMIONS IN AND OUT OF EQUILIBRIUM}

\section{A. Subsystem Rényi entropy, displacement operator, and trace formula}

In this section, we consider a system with fermionic degrees of freedom and derive a useful expansion of an arbitrary operator in terms of the so-called fermionic displacement operator [25] and show that the expansion can be used to represent Rényi entropies of a subregion of the system. A similar representation of Rényi entropy has been independently developed by Moitra and Sensarma [24]. The Rényi entropy of a quantum system described by a density matrix $\rho$ is obtained by dividing the system into two parts $A$ and $B$ (not necessarily equal) and defining a reduced density matrix,

$$
\rho_{A}=\operatorname{Tr}_{B} \rho
$$

for region $A$. If $\rho$ represents a pure state, then a measure of the quantum entanglement of region $A$ with $B$ can be obtained by evaluating the von Neumann entropy $S_{A}=-\operatorname{Tr}_{A}\left[\rho_{A} \ln \rho_{A}\right]$ by tracing over the degrees of freedom in $A$. In practice, however, the von Neumann entropy is often hard to calculate directly within field theoretic methods, and a more convenient measure of entanglement [1-4] is the $n$th Rényi entropy, $S_{A}^{(n)}$, defined as

$$
S_{A}^{(n)}=\frac{1}{1-n} \ln \operatorname{Tr}_{A}\left[\rho_{A}^{n}\right]
$$


where the integer $n>1$. The von Neumann entanglement entropy can be obtained by analytically continuing to $n \rightarrow 1$. We refer to the above subsystem Rényi entropy throughout the paper simply as Rényi entropy for brevity.

The main difficulty in evaluating the above comes from the representation of $\operatorname{Tr}_{A}\left[\rho_{A}^{n}\right]=\operatorname{Tr}_{A}\left[\left(\operatorname{Tr}_{B} \rho\right)\left(\operatorname{Tr}_{B} \rho\right) \ldots\left(\operatorname{Tr}_{B} \rho\right)\right]$ in a coherent-state path integral, since each factor of $\operatorname{Tr}_{B} \rho$ leads to separate replicas which need to be connected via appropriate boundary condition when represented through Grassmann variables [1,3]. To circumvent this difficulty, we derive the following operator expansion (see Appendix A) for an arbitrary operator $F$,

$$
F=\int d^{2}(\boldsymbol{\xi}, \boldsymbol{\eta}) f_{N}(\boldsymbol{\eta}, \boldsymbol{\xi}) \operatorname{Tr}\left[F D_{N}(\boldsymbol{\xi})\right] D_{N}(\boldsymbol{\eta}),
$$

where $\xi \equiv\left\{\bar{\xi}_{i}, \xi_{i}\right\}$ and $\boldsymbol{\eta} \equiv\left\{\bar{\eta}_{i}, \eta_{i}\right\}$ denote set of Grassmann variables with index $i=1, \ldots, N_{A}$, e.g., referring to a set of sites that includes the support of the operator $F$ on the lattice; $d^{2}(\boldsymbol{\xi}, \boldsymbol{\eta})=\prod_{i} d \bar{\xi}_{i} d \xi_{i} d \bar{\eta}_{i} d \eta_{i}$. Here the weight function $f_{N}(\boldsymbol{\xi}, \boldsymbol{\eta})$ is given by

$$
f_{N}(\boldsymbol{\eta}, \boldsymbol{\xi})=2^{N_{A}} \exp \left[-\frac{1}{2} \sum_{i}\left(\bar{\eta}_{i} \eta_{i}+\bar{\xi}_{i} \xi_{i}-\bar{\eta}_{i} \xi_{i}+\bar{\xi}_{i} \eta_{i}\right)\right] .
$$

The basis of above expansion in Eq. (3) is formed by the fermionic displacement operators [25], much like more familiar bosonic counterparts [22], defined as

$$
D(\xi)=\exp \left[\sum_{i}\left(c_{i}^{\dagger} \xi_{i}-\bar{\xi}_{i} c_{i}\right)\right]
$$

where $c_{i}^{\dagger}, c_{i}$ are the creation and annihilation operators on site $i$. The displacement operator generates the coherent state [25] $|\xi\rangle=D(\xi)|0\rangle$ by shifting the vacuum state $|0\rangle$ such that $c_{i}|\xi\rangle=\xi_{i}|\xi\rangle$. The displacement operator $D_{N}(\xi)$ that appears in Eq. (3) is normal ordered,

$$
\begin{aligned}
D_{N}(\boldsymbol{\xi}) & =\exp \left[\sum_{i} c_{i}^{\dagger} \xi_{i}\right] \exp \left[-\sum_{i} \bar{\xi}_{i} c_{i}\right] \\
& =D(\xi) \exp \left[\frac{1}{2} \sum_{i} \bar{\xi}_{i} \xi_{i}\right],
\end{aligned}
$$

and more conveniently used in the path-integral representation discussed in the next section.

Equation (3) offers a way to decompose a general operator $F$ using only the normal-ordered displacement operators $D_{N}(\xi)$ and is one of the key results of this paper. An important corollary to the decomposition identity of Eq. (3) is to express the trace of the product of operators $F$ and $G$ as

$$
\operatorname{Tr}[F G]=\int d^{2}(\boldsymbol{\xi}, \boldsymbol{\eta}) f_{N}(\boldsymbol{\xi}, \boldsymbol{\eta}) \operatorname{Tr}\left[F D_{N}(\boldsymbol{\xi})\right] \operatorname{Tr}\left[G D_{N}(\boldsymbol{\eta})\right]
$$

To derive the above, we have used the identities $\operatorname{Tr}\left[D_{N}(\boldsymbol{\eta}) G\right]=\operatorname{Tr}\left[G D_{N}(-\boldsymbol{\eta})\right] \quad$ and $\quad f_{N}(-\boldsymbol{\eta}, \boldsymbol{\xi})=f_{N}(\boldsymbol{\xi}, \boldsymbol{\eta})$ (see Appendix A). Equation (7) is the second key result of this paper and is crucial for deriving a path-integral representation for evaluating Rényi entropy as we discuss in the next section. We could also make the operator expansion $F=\int d^{2}(\xi, \boldsymbol{\eta}) f(\boldsymbol{\eta}, \boldsymbol{\xi}) \operatorname{Tr}[F D(\xi)] D(\boldsymbol{\eta})$ and obtain the corresponding trace formula similar to Eq. (7), in terms of the displacement operator $D(\xi)$ and weight function $f(\boldsymbol{\eta}, \boldsymbol{\xi})=2^{N_{A}} \exp \left[\sum_{i}\left(\bar{\eta}_{i} \xi_{i}-\bar{\xi}_{i} \eta_{i}\right) / 2\right]$.

Using the trace formula [Eq. (7)], the second-Rényi entropy, $S_{A}^{(2)}$, can be conveniently expressed as

$$
\begin{aligned}
e^{-S_{A}^{(2)}} & =\operatorname{Tr}_{A}\left[\rho_{A} \rho_{A}\right] \\
& =\int d^{2}(\boldsymbol{\xi}, \boldsymbol{\eta}) f_{N}(\boldsymbol{\xi}, \boldsymbol{\eta}) \operatorname{Tr}_{A}\left[\rho_{A} D_{N}(\boldsymbol{\xi})\right] \operatorname{Tr}_{A}\left[\rho_{A} D_{N}(\boldsymbol{\eta})\right]
\end{aligned}
$$

In the above, $\xi \equiv\left\{\bar{\xi}_{i}, \xi_{i}\right\}_{i \in A}$ (and similarly for $\eta$ ); i.e., the displacements operators above only involve the fermionic operators in the region $A$. As a result, we have

$$
\operatorname{Tr}_{A}\left[\rho_{A} D_{N}(\xi)\right]=\operatorname{Tr}\left[\rho D_{N}(\xi \in A)\right] ;
$$

i.e., the expectation value of the operator $D(\xi)$ evaluated for the reduced system is same as that obtained using the full density matrix. Equation (9), therefore, eliminates the need to calculate the reduced density matrix $\rho_{A}$. The evaluation of $\rho_{A}$ is a difficult step for calculating entanglement entropy, as mentioned earlier. To proceed further, we define the normalordered fermionic Wigner characteristic function [22,25] for the density matrix,

$$
\chi_{N}(\xi)=\operatorname{Tr}\left[\rho D_{N}(\xi \in A)\right],
$$

and arrive at the final expression for the second-Rényi entropy for region $A$ :

$$
e^{-S_{A}^{(2)}}=\int_{\xi, \boldsymbol{\eta} \in A} d^{2}(\boldsymbol{\xi}, \boldsymbol{\eta}) f_{N}(\boldsymbol{\xi}, \boldsymbol{\eta}) \chi_{N}(\boldsymbol{\xi}) \chi_{N}(\boldsymbol{\eta}) .
$$

We also sometime use an analogous expression written in terms of $D(\xi)$ by replacing $D_{N}(\xi)$ and the function $f_{N}(\boldsymbol{\xi}, \boldsymbol{\eta})$ by $f(\xi, \eta)$. This leads to the usual fermionic characteristic function [25],

$$
\chi(\xi)=\operatorname{Tr}[\rho D(\xi \in A)] .
$$

The higher order Rényi entropies, $S_{A}^{(n>2)}$, can be found in a similar manner by repeated application of the trace identity in Eq. (7). In fact, as we show in Sec. II C, a hierarchy for higher order Rényi entropies can be derived that recursively expresses the characteristic function of higher moments of the reduced density matrix $\rho_{A}$ in terms of the lower order ones.

\section{B. Equilibrium and nonequilibrium field theories for Rényi entropy}

A path-integral representation of the characteristic function, $\chi_{N}(\xi)$, will naturally lead to a similar representation for the second Rényi entropy of region $A$ through Eq. (11). Therefore, we first derive the path integral for $\chi_{N}(\xi)$ for (a) thermal density matrix describing a system in equilibrium, and (b) time-evolving density matrix subjected to a general time-dependent Hamiltonian, e.g., describing a quench.

\section{Path integral for thermal equilibrium}

The density matrix for a system described by a Hamiltonian $H$ under thermal equilibrium is given by

$$
\rho=Z^{-1} \exp [-\beta H],
$$


with the partition function

$$
Z=\operatorname{Tr}[\exp (-\beta H)]
$$

and inverse temperature $\beta=1 / T\left(k_{\mathrm{B}}=1\right)$. Inserting the identity operator $\int d^{2} c|c\rangle\langle c|=I$ in the coherent state basis, we get

$$
\chi_{N}(\xi)=Z^{-1} \int \prod_{n=0}^{1} d^{2} c_{n}\left\langle-c_{0}\left|e^{-\beta H}\right| c_{1}\right\rangle\left\langle c_{1}\left|D_{N}(\xi)\right| c_{0}\right\rangle,
$$

with $d^{2} c_{n}=\prod_{i} d \bar{c}_{\text {in }} d c_{i n}$.
Using Eq. (6), the matrix element for the displacement operator is easily evaluated as

$$
\left\langle c_{1}\left|D_{N}(\xi)\right| c_{0}\right\rangle=\exp \left(\sum_{i \in A} \bar{c}_{i, 1} \xi_{i}-\bar{\xi}_{i} c_{i, 0}\right)\left\langle c_{1} \mid c_{0}\right\rangle .
$$

Finally, following the standard methodology [36] of fermionic coherent state, e.g., evaluating $\left\langle-c_{0}\left|e^{-\beta H}\right| c_{1}\right\rangle$ via Trotter decomposition $\beta=N_{\tau} \Delta \tau$ and taking the continuum limit $N_{\tau} \rightarrow \infty, \Delta \tau \rightarrow 0$, we get

$$
\chi_{N}(\xi)=Z^{-1} \int \mathcal{D}(\bar{c}, c) \exp \left[-\int_{0}^{\beta} d \tau\left\{\sum_{i} \bar{c}_{i}(\tau) \partial_{\tau} c_{i}(\tau)+H\left(\bar{c}_{i}(\tau), c_{i}(\tau)\right)-\sum_{i \in A} \bar{c}_{i}(\tau) \delta\left(\tau^{+}\right) \xi_{i}+\sum_{i \in A} \bar{\xi}_{i} \delta(\tau) c_{i}(\tau)\right\}\right],
$$

as the path-integral representation of the characteristic function. The above equation offers a significant advantage over the usual field-theoretic formalism [1-3] used to compute Rényi entropy. The imaginary-time boundary condition for the Grassmann fields $c_{i}(\tau)$ and $\bar{c}_{i}(\tau)$ are still antiperiodic, i.e., $c_{i}(\tau+\beta)=-c_{i}(\tau)$, irrespective of whether $i$ belongs to the region $A$. Instead, the distinction between subsystem $A$ and the rest of the system is encoded by the auxiliary fields $\xi$ which only couple with the fields $c_{i}(\tau)$ and $\bar{c}_{i}(\tau)$ for $i \in A$. Under time discretization, relevant for the numerical implementation discussed later, we have

$$
\begin{aligned}
\bar{c}_{i}(\tau) \partial_{\tau} c_{i}(\tau) & =\bar{c}_{i, n} \frac{\left(c_{i, n}-c_{i, n-1}\right)}{\Delta \tau} \\
\delta\left(\tau^{+}\right) & =\frac{1}{\Delta \tau} \delta_{n, 1}, \quad \delta(\tau)=\frac{1}{\Delta \tau} \delta_{0, n},
\end{aligned}
$$

where $n$ denotes the $n$th time index.

\section{Path integral for nonequilibrium evolution}

The density matrix, at a time $t$, evolving under a timedependent Hamiltonian $H(t)$ is given by

$$
\rho(t)=U\left(t, t_{0}\right) \rho_{0} U\left(t_{0}, t\right),
$$

where $\rho_{0}$ is the initial density matrix at time $t_{0}$. The operator $U\left(t_{1}, t_{2}\right)$ is the unitary evolution operator associated with the Hamiltonian $H(t)$ and defined as

$$
U\left(t_{1}, t_{2}\right)=\left\{\begin{array}{ll}
\mathrm{T}\left[\exp \left(-i \int_{t_{1}}^{t_{2}} d t H(t)\right)\right] & t_{1} \geqslant t_{2} \\
\tilde{\mathrm{T}}\left[\exp \left(-i \int_{t_{1}}^{t_{2}} d t H(t)\right)\right] & t_{1}<t_{2}
\end{array},\right.
$$

where $\mathrm{T}$ and $\tilde{\mathrm{T}}$ are the time-ordering and anti-time-ordering operators respectively. We use Schwinger-Keldysh closed time-contour formalism $[37,38]$ to obtain a path-integral representation for the Rényi entropy, e.g., $S^{(2)}(t)$, which is now time dependent and given by

$$
e^{-S_{A}^{(2)}(t)}=\int_{\xi, \eta \in A} d^{2}(\xi, \eta) f_{N}(\boldsymbol{\xi}, \boldsymbol{\eta}) \chi_{N}(\xi, t) \chi_{N}(\boldsymbol{\eta}, t)
$$

Here we have rewritten the trace identity [Eq. (7)] (see Appendix A) to define the time-dependent characteristic function as

$$
\chi_{N}(\boldsymbol{\xi}, t)=\operatorname{Tr}\left[D_{N}(\boldsymbol{\xi}) \rho(t)\right]=\operatorname{Tr}\left[U\left(t_{0}, t\right) D_{N}(\boldsymbol{\xi}) U\left(t, t_{0}\right) \rho_{0}\right],
$$

for the sake of convenience in constructing the path-integral representation.

As in the standard Schwinger-Keldysh closed-time contour formalism [37,38], the last line in Eq. (22) may be interpreted, from right to left, as starting from an initial density matrix $\rho_{0}$, evolving forward in time [represented by + branch in Fig. 1(a)] by $U\left(t, t_{0}\right)$ from $t_{0}$ to $t$, applying fermionic source fields $\xi$ in region $A$, through $D_{N}(\xi)$, at time $t$ and then going back to $t_{0}$ via the backward time-evolution [- branch in Fig. 1(a)] $U\left(t_{0}, t\right)$. We refer to the closed-time contour with the symbol $\mathcal{C}$ and use a contour variable $z$, which takes values $(t,+),(t,-)$ at time $t$ for the,+- branches. As done often in the Schwinger-Keldysh formalism, the contour is extended to $+\infty$ [see Fig. 1]. We incorporate this contour extension in our expression for the characteristic function:

$$
\chi_{N}(\xi, t)=\operatorname{Tr}\left[U\left(t_{0}, t\right) U(t, \infty) U(\infty, t) D_{N}(\xi) U\left(t, t_{0}\right) \rho_{0}\right] .
$$

Again following standard route [36,37], as in the thermal equilibrium case, we obtain a path-integral representation for

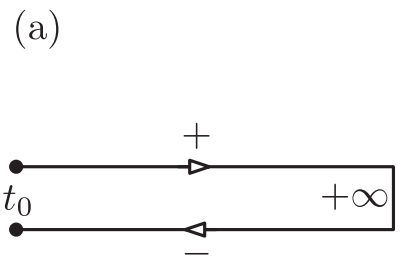

(b)

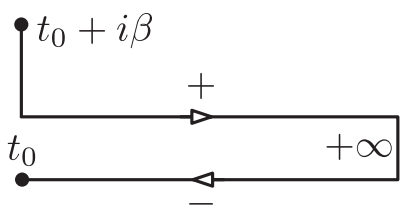

FIG. 1. Closed time contours (CTCs): (a) The SchwingerKeldysh contour, for an arbitrary initial density matrix $\rho_{0}$, starting at time $t_{0}$ extending to $+\infty$ and returning back to $t_{0}$. The two branches represent the forward (+) and backward (-) evolution of time respectively. (b) The modified contour for an initial density matrix picked from a thermal ensemble, i.e., $\rho_{0} \sim \exp (-\beta H)$. The $\exp (-\beta H)$ term is incorporated into the contour as an evolution in imaginary time, represented by the additional vertical branch of length $\beta$. 
$\chi_{N}(\xi, t)$, in terms of the entanglement Keldysh action:

$$
\begin{aligned}
\mathcal{S}^{\mathcal{C}}= & \int_{\mathcal{C}} d z\left[\sum_{i} \bar{c}_{i}(z) i \partial_{z} c_{i}(z)-H(\bar{c}(z), c(z))\right] \\
& -i \int_{\mathcal{C}} d z \sum_{i \in A}\left[\bar{c}_{i}(z) \delta_{\mathcal{C}}\left(z,\left(t^{+},+\right)\right) \xi_{i}\right. \\
& \left.-\bar{\xi}_{i} \delta_{\mathcal{C}}(z,(t,+)) c_{i}(z)\right],
\end{aligned}
$$

such that

$$
\chi_{N}(\xi, t)=\int \mathcal{D}(\bar{c}, c) e^{i \mathcal{S}^{\mathcal{C}}}\left\langle c_{i}(0,+)\left|\rho_{0}\right|-c_{i}(0,-)\right\rangle .
$$

Here $\delta_{\mathcal{C}}\left(z, z_{1}\right)$ is the Dirac $\delta$ function on $\mathcal{C}$ and takes into account both the time argument and the branch index $( \pm)$. The coherent-state matrix element of the initial density matrix, $\left\langle c_{i}(0,+)\left|\rho_{0}\right|-c_{i}(0,-)\right\rangle$ in Eq. (25), encodes the information of the initial state at starting time $t_{0}$. For a thermal initial state, one can add an additional branch to $\mathcal{C}$ and redefine it as

$$
\mathcal{C}=\left[t_{0}+i \beta, t_{0}\right) \cup\left[t_{0},+\infty\right) \cup\left(+\infty, t_{0}\right] ;
$$

i.e., the contour is now composed of a vertical imaginary-time branch of length $\beta$ and the usual + and - branches, as shown in Fig. 1(b). The form of the action $\mathcal{S}^{\mathcal{C}}$ remains exactly the same as given in Eq. (24) such that

$$
\chi_{N}(\xi, t)=Z_{0}^{-1} \int \mathcal{D}(\bar{c}, c) e^{i \mathcal{S}^{c}},
$$

where $Z_{0}$ is the partition function for the initial thermal state described by $\rho_{0}$. We emphasize here that the Hamiltonian describing the $\rho_{0}$ and the one dictating the unitary evolution [see Eq. (20)] do not need to be same, e.g., in a quench [39-42]. This distinction is incorporated by choosing the appropriate Hamiltonian in Eq. (24) for the imaginary-time and real-time branches.

In the next section, we show that the equilibrium and nonequilibrium coherent-state path integrals can be used immediately to obtain well-known expressions for Rényi and von Neumann entanglement entropies in terms of the correlation matrix [3].

\section{Rényi entropies for noninteracting fermions}

We consider a noninteracting system described by the thermal density matrix

$$
\rho=\exp \left[-\beta \sum_{i j} t_{i j} c_{i}^{\dagger} c_{j}\right] / Z
$$

where $Z=\operatorname{Tr} \exp \left[-\beta \sum_{i j} t_{i j} c_{i}^{\dagger} c_{j}\right]$ is the partition function. Using Eq. (17), the normal-ordered characteristic function in this case is given by

$$
\chi_{N}(\xi)=Z^{-1} \int \mathcal{D}(\bar{c}, c) \exp \left[-\int_{0}^{\beta} d \tau\left\{\sum_{i j} \bar{c}_{i}(\tau)\left(\delta_{i j} \partial_{\tau}+t_{i j}\right) c_{j}(\tau)-\sum_{i \in A} \bar{c}_{i}(\tau) \delta\left(\tau^{+}\right) \xi_{i}+\sum_{i \in A} \bar{\xi}_{i} \delta(\tau) c_{i}(\tau)\right\}\right]
$$

The Gaussian structure of the fermionic integral allows us to integrate the fermions to give

$$
\chi_{N}(\xi)=\exp \left[-\int d \tau_{1,2} \sum_{i j} \bar{\xi}_{i} \delta\left(\tau_{1}\right)(-G)_{i j}\left(\tau_{1}, \tau_{2}\right) \delta\left(\tau_{2}^{+}\right) \xi_{j}\right],
$$

where the matrix $G$, is the Green's function and is defined as the imaginary-time-ordered two point correlator

$$
\begin{aligned}
G_{i j}\left(\tau_{1}, \tau_{2}\right) & =-\left\langle T_{\tau} c_{i}\left(\tau_{1}\right) c_{j}^{\dagger}\left(\tau_{2}\right)\right\rangle \\
& = \begin{cases}-\left\langle c_{i}\left(\tau_{1}\right) c_{j}^{\dagger}\left(\tau_{2}\right)\right\rangle & \tau_{1}>\tau_{2} \\
+\left\langle c_{j}^{\dagger}\left(\tau_{2}\right) c_{i}\left(\tau_{1}\right)\right\rangle & \tau_{1}<\tau_{2}\end{cases}
\end{aligned}
$$

and can be calculated using

$$
\left(-G^{-1}\right)_{i j}\left(\tau_{1}, \tau_{2}\right)=\left(\delta_{i j} \partial_{\tau}+t_{i j}\right) \delta\left(\tau_{1}-\tau_{2}\right) .
$$

However, because of the $\delta$ functions in Eq. (30), we need to evaluate only $G_{i j}\left(0,0^{+}\right)$. This allows us to define a correlation matrix [3] $C_{i j}$, as follows:

$$
C_{i j}^{T}=C_{j i} \equiv G_{i j}\left(0,0^{+}\right)=\left\langle c_{j}^{\dagger} c_{i}\right\rangle=\operatorname{Tr}\left[\rho c_{j}^{\dagger} c_{i}\right],
$$

where $C^{T}$ is transpose of the matrix $C$. Therefore, the characteristic functions for an arbitrary noninteracting system are given by

$$
\begin{aligned}
\chi_{N}(\xi) & =\exp \left[\sum_{i j \in A} \bar{\xi}_{i} C_{i j}^{T} \xi_{j}\right], \\
\chi(\xi) & =\exp \left[\sum_{i j \in A} \bar{\xi}_{i} C_{i j}^{T} \xi_{j}-\frac{1}{2} \sum_{i \in A} \bar{\xi}_{i} \xi_{i}\right] .
\end{aligned}
$$

The Gaussian structure of the characteristic function is a direct consequence of the underlying noninteracting Hamiltonian. Using Eq. (11) and the characteristic function above, the second Rényi entropy can be immediately evaluated by integrating out the auxiliary Grassmann variables $\xi$ and $\boldsymbol{\eta}$ to get the well-known expression [3] for noninteracting fermions,

$$
S^{(2)}=-\operatorname{Tr} \ln \left[(\mathbf{1}-C)^{2}+C^{2}\right],
$$

where $\mathbf{1}$ is a $N_{A} \times N_{A}$ identity matrix and only the elements $C_{i j}$ of the correlation matrix [see Eq. (33)] with $i, j \in A$ are involved in the above expression. In fact, the Gaussian form of the characteristic functions allows us to derive the expression for the higher Rényi entropies as well. To this end, we first define the higher order generalization of the characteristic function [see Eq. (10)]

$$
\chi_{n}(\xi)=\operatorname{Tr}_{A}\left[\rho_{A}^{n} D(\xi \in A)\right] .
$$


A recursion relation of the form

$$
\chi_{n+1}(\boldsymbol{\alpha})=\frac{1}{2^{N_{A}}} \int d^{2}(\boldsymbol{\xi}, \boldsymbol{\eta}) f(\boldsymbol{\xi}, \boldsymbol{\eta}) f(\boldsymbol{\eta}, \boldsymbol{\alpha}) \chi_{n}(\boldsymbol{\xi}) \chi_{1}(\boldsymbol{\alpha}+\boldsymbol{\eta}),
$$

with $\chi_{n=1}=\chi$, can then be derived (see Appendix B 1), which obtains higher order $\chi$ from lower ones. As alluded earlier, we use a Gaussian ansatz of the form

$$
\chi_{n}(\boldsymbol{\alpha})=\lambda_{n} \exp \left[\bar{\alpha}^{T} A_{n} \alpha\right],
$$

where $\alpha=\left[\begin{array}{lll}\alpha_{1} & \ldots & \alpha_{N_{A}}\end{array}\right]^{T}$ and $A_{n}$ is a $N_{A} \times N_{A}$ complex matrix, to solve the recursion. The ansatz is consistent with the expression for $\chi$ obtained in Eq. (35), provided we set

$$
\begin{aligned}
& A_{1}=A=C^{T}-\mathbf{1} / 2, \\
& \lambda_{1}=\lambda=1 .
\end{aligned}
$$

The ansatz lets us simplify the recursion (see Appendix B 2) and derive recursions involving $\lambda_{n}$ and $A_{n}$, i.e.,

$$
\begin{aligned}
\lambda_{n+1} & =\operatorname{det}\left[2 A_{n} A+\mathbf{1} / 2\right] \lambda_{n}, \\
A_{n+1} & =\left(A+A_{n}\right)\left(\mathbf{1}+4 A A_{n}\right)^{-1} .
\end{aligned}
$$

The last equation can be rearranged (see Appendix B 2) into

$$
X_{n+1}=\mathbf{1}+C^{T}\left(C^{T}-\mathbf{1}\right) X_{n}{ }^{-1},
$$

with $X_{n}$ defined as

$$
X_{n}=2 A_{n} A+\frac{1}{2} .
$$

We find the following

$$
X_{n}=\left[\left(\mathbf{1}-C^{T}\right)^{n}+\left(C^{T}\right)^{n}\right]\left[\left(\mathbf{1}-C^{T}\right)^{n-1}+\left(C^{T}\right)^{n-1}\right]^{-1}
$$

solves Eq. (43); see Appendix B 3. The $n$th Rényi entropy is obtained by evaluating $\lambda_{n}$, since

$$
\chi_{n}(\boldsymbol{\alpha}=0)=\operatorname{Tr}_{A}\left[\left(\rho_{A}\right)^{n} D(\boldsymbol{\alpha}=0)\right]=\operatorname{Tr}_{A}\left[\left(\rho_{A}\right)^{n}\right]=\lambda_{n},
$$

and Eq. (2) implies

$$
S_{A}^{(n)}=\frac{1}{1-n} \ln \operatorname{Tr}_{A}\left[\left(\rho_{A}\right)^{n}\right]=\frac{1}{1-n} \ln \lambda_{n} .
$$

From the recursion for $\lambda_{n}$ in Eq. (42), and Eq. (44), we see that

$$
\lambda_{n}=\operatorname{det}\left(X_{n}\right) \lambda_{n-1}=\operatorname{det}\left(X_{n} X_{n-1} \ldots X_{1}\right) .
$$

Looking at the structure of $X_{n}$ in Eq. (45), we find that the inverse in $X_{n}$ cancels with the noninverse term in $X_{n-1}$, and so forth, leaving at the end

$$
\begin{aligned}
\operatorname{Tr}_{A}\left[\rho_{A}^{n}\right]=\lambda_{n} & =\operatorname{det}\left[\left(\mathbf{1}-C^{T}\right)^{n}+\left(C^{T}\right)^{n}\right] \\
& =\operatorname{det}\left[(\mathbf{1}-C)^{n}+C^{n}\right],
\end{aligned}
$$

from which we find the $n$th Rényi entropy to be

$$
S_{A}^{(n)}=\frac{1}{1-n} \operatorname{Tr}\left[\ln \left[(\mathbf{1}-\boldsymbol{C})^{n}+\boldsymbol{C}^{n}\right]\right],
$$

in agreement with the known results [3]. Taking the limit $n \rightarrow 1$ above, we get the subsystem von Neumann entropy, $S_{\mathrm{EE}}=-\operatorname{Tr}[(\mathbf{1}-C) \ln (\mathbf{1}-C)+C \ln C]$.

As shown in Appendix $C$, in the nonequilibrium case, using the Schwinger-Keldysh path integral, discussed in Sec. II B 2, we obtain the same expression for the $n$, the Rényi entropy
$S_{A}^{(n)}(t)$, as given in Eq. (50) with a time-dependent correlation matrix

$$
C_{i j}(t)=\operatorname{Tr}\left[\rho(t) c_{i}^{\dagger} c_{j}\right]
$$

Here $\rho(t)$ is the density matrix at time $t$.

Having reproduced the results for noninteracting fermionic systems, we now seek to set up the field theory for Rényi entropy in some well-known models for interacting fermions in the next sections. We discuss the formulation for two types of systems, large- $N$ fermionic models based on the SYK model $[27,28]$ and a repulsive Hubbard model treated within single-site DMFT [29].

\section{Rényi entropy for SYK and related models}

SYK model [27,28] and its various extensions [30,32,4349] have emerged in recent years as a major paradigm to study phases of strongly interacting fermions, e.g., NFL, marginal and heavy Fermi liquids, strong-coupling superconductivity [32,46-51], and quantum phase transitions [30,48,49,52,53]. Remarkably, these models can be solved exactly in a suitable large- $N$ limit, without resorting to any kind of perturbative treatment. As a result, the study of this family of large- $N$ fermionic model has given insights into transport [32,4347], thermalization [54-62], many-body chaos [27,30,34], and entanglement $[43,63-67]$ in strongly interacting fermionic systems, as well as their intriguing connections with black holes $[27,28,35]$. Here we use the path-integral technique of Sec. II B to formulate the field theory for the second Rényi entropy for the SYK model and its various generalizations discussed below.

\section{SYK model for non-Fermi liquid state}

The SYK model describes a zero-dimensional system having $N$ fermion flavors or sites [see Fig. 2(a)] that interact via an all-to-all or infinite-range Hamiltonian. To keep the discussion general, we use the $q$-body version [35] of the SYK model, $\mathrm{SYK}_{q}$ (typically referred in the literature as $\mathrm{SYK}_{2 q}$ ) described by the Hamiltonian

$$
H_{S Y K_{q}}=\sum_{i_{1} \ldots i_{q} ; j_{1} \ldots j_{q}} J_{i_{1} \ldots i_{q} ; j_{q} \ldots j_{1}} c_{i_{1}}^{\dagger} \ldots c_{i_{q}}^{\dagger} c_{j_{q}} \ldots c_{j_{1}}
$$

where $J_{i_{1} \ldots i_{q} ; j_{q} \ldots j_{1}}$ are properly antisymmetrized Gaussian random numbers with variance $J^{2} / q N^{2 q-1}(q !)^{2}$. Setting $q=2$ in the above Hamiltonian gives back the original SYK model $[27,28]$. The ground state for the $\mathrm{SYK}_{q}$ model, for $q \geqslant 2$, has been shown to be a non-Fermi liquid (NFL), lacking quasiparticle excitations, with a fermion scaling dimension $\Delta=1 / 2 q$ [35]. Interestingly, the SYK NFL states possess a residual zero-temperature thermodynamic entropy $S_{0}$ in the limit $N \rightarrow \infty$, e.g., $S_{0} \approx 0.464$ for $q=2$, which, as we shall see later, plays a crucial role in the interpretation of the $T=0$ Rényi entropy in the large- $N$ limit as well. The $q=1$ model is a special case whose ground state is a noninteracting Fermi liquid described by a single-particle semicircular density of states (DOS). 


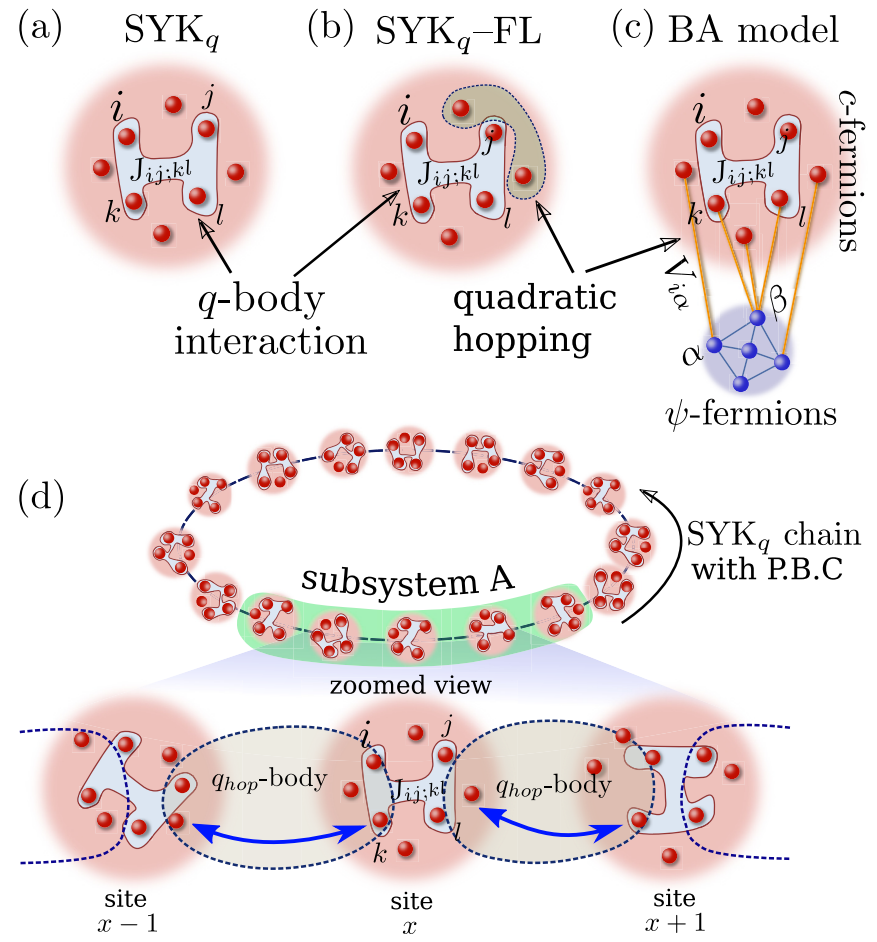

FIG. 2. Large- $N$ models: (a) The $q$-body SYK model $\left(\mathrm{SYK}_{q}\right)$ for complex fermions shown for $q=2$ interactions that scatter a pair of fermions, e.g., from sites $k, l$ to sites $i, j$ with a random complex amplitude $J_{i j k l}$. (b) Model for a zero-dimensional interacting Fermi liquid obtained from the $\mathrm{SYK}_{q}$ model by adding quadratic (onebody) hopping terms (represented by dashed lines) on top of the $q$-body $(q \geqslant 2)$ interactions. (c) The Banerjee-Altman (BA) model for NFL-FL transition, obtained by joining a noninteracting dot of peripheral $\psi$ fermions (blue dots joined by lines) to the SYK dot of $c$ fermions via random hopping amplitudes $V_{i \alpha}$. (d) A chain of $\mathrm{SYK}_{q}$ dots with periodic boundary conditions (PBC). Each dot is connected to its nearest neighbors via $q_{\text {hop }}$-body terms, e.g., $q_{\text {hop }}=1$, as shown in the enlarged view (below). The highlighted part of the chain is chosen as the subsystem $A$ for which entanglement entropy is calculated.

\section{SYK model with quadratic hopping term for Fermi liquid state}

The SYK model has also been generalized to describe strongly interacting or heavy Fermi liquids (FL) [32] by adding a random all-to-all hopping $(q=1)$ term to Eq. (52), i.e.,

$$
H_{\mathrm{FL}}=\sum_{i j} t_{i j} c_{i}^{\dagger} c_{j}+\sum_{i_{1} \ldots i_{q} ; j_{1} \ldots j_{q}} J_{i_{1} \ldots i_{q} ; j_{q} \ldots j_{1}} c_{i_{1}}^{\dagger} \ldots c_{i_{q}}^{\dagger} c_{j_{q}} \ldots c_{j_{1}},
$$

where $t_{i j}$ are the Gaussian random numbers with variance $t_{\text {hop }}^{2} / N$ [see Fig. 2(b)].

\section{BA model for non-Fermi liquid to Fermi liquid transition}

We also study Rényi entropy in the Banerjee-Altman (BA) model of Ref. [30], $\mathcal{H}=\mathcal{H}_{c}+\mathcal{H}_{\psi}+\mathcal{H}_{c \psi}$, where

$$
\mathcal{H}_{c}=\sum_{i j k l} J_{i j k l} c_{i}^{\dagger} c_{j}^{\dagger} c_{k} c_{l}
$$

$$
\begin{aligned}
\mathcal{H}_{\psi} & =\sum_{\alpha \beta} t_{\alpha \beta} \psi_{\alpha}^{\dagger} \psi_{\beta}, \\
\mathcal{H}_{c \psi}(t) & =\sum_{i \alpha}\left(V_{i \alpha} c_{i}^{\dagger} \psi_{\alpha}+V_{i \alpha}^{*} \psi_{\alpha}^{\dagger} c_{i}\right) .
\end{aligned}
$$

The above model [see Fig. 2(c)] has two species of fermions: (1) the SYK fermions $(c)$, on sites $i=1, \ldots, N_{c}$, interacting with random coupling $J_{i j k l}$ [Eq. (54a)] with variance $J^{2} /\left(2 N_{c}\right)^{3 / 2}$, and (2) the peripheral fermions $(\psi)$, on a separate set of sites $\alpha=1, \ldots, N_{\psi}$ connected via random all-to-all hopping $t_{\alpha \beta}$ [Eq. (54b)]. The SYK and the peripheral fermions are quadratically coupled via $V_{i \alpha} ; t_{\alpha \beta}$ and $V_{i \alpha}$ are Gaussian random variables with variances $t_{\text {hop }}^{2} / N_{c}$ and $V^{2} / \sqrt{N_{c} N_{\psi}}$, respectively.

The model is exactly solvable for $N_{c}, N_{\psi} \rightarrow \infty$ with a fixed ratio $p=N_{\psi} / N_{c}$, that is varied to go through the QPT between NFL and FL at a critical value $p=p_{c}=1$ [30]. The residual entropy density $S_{0}(p)$ of the SYK NFL continuously vanishes at the transition [30].

\section{Lattice of SYK dots for interacting diffusive metal}

Extension of the above zero-dimensional models to higher dimensions have also been achieved [31,32,45,47,48]. These systems typically involve a lattice of $\mathrm{SYK}_{q}$ dots, each having $N$ fermion flavors, connected to their nearest neighbors via $q_{\text {hop-body terms and can be described using the general }}$ Hamiltonian

$$
\begin{aligned}
H= & \sum_{\left\langle x x^{\prime}\right\rangle i_{1} \ldots i_{q} ; j_{1} \ldots j_{q}} t_{x, i_{1} \ldots i_{q} ; x^{\prime} j_{q} \ldots j_{1}} c_{x i_{1}}^{\dagger} \ldots c_{x i_{q}}^{\dagger} c_{x^{\prime} j_{q}} \ldots c_{x^{\prime} j_{1}}+\text { H.c. } \\
& +\sum_{x, i_{1} \ldots i_{q} ; j_{1} \ldots j_{q}} J_{x, i_{1} \ldots i_{q} ; j_{q} \ldots j_{1}} c_{x i_{1}}^{\dagger} \ldots c_{x i_{q}}^{\dagger} c_{x j_{q}} \ldots c_{x j_{1}},
\end{aligned}
$$

where the coordinates $x, x^{\prime}$ denote the position of the individual dot in the lattice or chain and $\left\langle x x^{\prime}\right\rangle$ represents the nearest neighbors [see Fig. 2(d)]. The amplitude $t_{x x^{\prime}}$ and $J_{x}$ are independent Gaussian random numbers with variances having the same form as that of the coupling $J$ in Eq. (52); $q \rightarrow q_{\text {hop }}$ and $J \rightarrow t_{\text {chain }}$ for the $t_{x x^{\prime}}$ amplitudes. Setting $q_{\text {hop }}=q=1$ produces a noninteracting diffusive metal, while $q_{\text {hop }}=1$, $q=2$ and $q_{\text {hop }}=2, q=2$ result in a diffusive heavy Fermi liquid [32] or a non-Fermi liquid [43], respectively.

\section{Rényi entropy in the thermal state}

Here we derive the exact equations to evaluate the Rényi entropy for all the models of the preceding sections at the large- $N$ limit in thermal equilibrium. The evaluation of Rényi entropy for nonequilibrium evolution can be performed using the Schwinger-Keldysh path integral formalism of Sec. II B 2 and is discussed in Appendix D. In order to access the second Rényi entropy at zero temperature, we use the thermal density matrix to perform the analysis and then take the $T \rightarrow 0$ limit. The definition for second Rényi entropy [see Eq. (2)] implies that we need to calculate the disorder averaged quantity

$$
S^{(2)}=-\overline{\ln \left(\operatorname{Tr}_{A}\left[\rho_{A}^{2}\right]\right)}=-\overline{\ln \operatorname{Tr}_{A}\left[Z_{A}^{2}\right]}+2 \overline{\ln Z},
$$


where $\cdots$ represents disorder average over the amplitudes $J_{i_{1} \ldots i_{q} ; j_{q} \ldots j_{1}}$. We also define the operator $Z_{A}=\operatorname{Tr}_{B} \exp (-\beta H)$. We evaluate each term in Eq. (56) separately. Using the trace identity [see Eq. (7)] we formally write down $\operatorname{Tr}_{A}\left[Z_{A}^{2}\right]$ as

$$
\operatorname{Tr}_{A}\left[Z_{A}^{2}\right]=\int d^{2}(\boldsymbol{\xi}, \boldsymbol{\eta}) f_{N}(\boldsymbol{\xi}, \boldsymbol{\eta}) \operatorname{Tr}\left[Z D_{N}(\boldsymbol{\xi})\right] \operatorname{Tr}\left[Z D_{N}(\boldsymbol{\eta})\right],
$$

where as before $\xi$ and $\eta$ couple only to sites in the region $A$, and $\operatorname{Tr}\left[Z D_{N}(\xi)\right]$ is the characteristic function for the partition function $Z$. Since we need to evaluate the logarithm of $\operatorname{Tr}_{A}\left[Z_{A}^{2}\right]$ [see Eq. (56)], we use the replica trick to express the logarithm as a product of disorder replicas

$$
\overline{\ln \operatorname{Tr}_{A}\left[Z_{A}^{2}\right]}=\lim _{r \rightarrow 0} \frac{\overline{\operatorname{Tr}_{A}\left[Z_{A}^{2}\right]^{r}}-1}{r} .
$$

The replicas, along with Eq. (57) and the integral representation in Eq. (17), allow us to write the path integral for the $\mathrm{SYK}_{q}$ model [Eq. (52)]

$$
\begin{aligned}
\operatorname{Tr}_{A}\left[Z_{A}^{2}\right]^{r}=2^{N_{A} r} & \int \mathcal{D}(\bar{c}, c) d^{2}(\boldsymbol{\xi}, \boldsymbol{\eta}) \exp \left[-\int_{0}^{\beta} d \tau \sum_{i, \sigma, a=1}^{r} \bar{c}_{i \sigma a} \partial_{\tau} c_{i \sigma a}+\sum_{i \in A, \sigma, a} \int_{0}^{\beta} d \tau\left[\delta\left(\tau^{+}\right) \bar{c}_{i \sigma a}(\tau) \xi_{i \sigma a}-\bar{\xi}_{i \sigma a} \delta(\tau) c_{i \sigma a}(\tau)\right]\right. \\
& \left.-\int_{0}^{\beta} d \tau \sum_{i, j, \sigma, a} J_{i_{1} \ldots i_{q} ; j_{q} \ldots j_{1}} \bar{c}_{i_{1} \sigma a} \ldots \bar{c}_{i_{q} \sigma a} c_{j_{q} \sigma a} \ldots c_{j_{1} \sigma a}\right] \exp \left[\frac{1}{2} \sum_{i \in A, a}\left(\bar{\xi}_{i a} \eta_{i a}-\bar{\eta}_{i a} \xi_{i a}-\bar{\xi}_{i a} \xi_{i a}-\bar{\eta}_{i a} \eta_{i a}\right)\right] .
\end{aligned}
$$

The Grassmann fields $\bar{c}_{i \sigma a}(\tau), c_{i \sigma a}(\tau)$, appearing in the path integral above and representing the fermions in the model, are labeled by several indices apart from the site indices $i, j=1, \ldots, N$-an imaginary-time coordinate $\tau$, a disorder replica index $a=1, \ldots r$, and an entanglement replica index $\sigma=1,2$. Here $\xi_{i 1 a}=\xi_{i a}$ and $\xi_{i 2 a}=\eta_{i a}$, indicating the Grassmann field originates either from the characteristic function $\operatorname{Tr}[Z D(\xi)]$ or $\operatorname{Tr}[Z D(\eta)]$, respectively in Eq. (57). The Grassmann variables $\xi$ and $\eta$ have the same indices as $\bar{c}, c$ except for the time coordinate $\tau$. The subsystem or the region $A$ in the zero-dimensional model is defined as any of the $N_{A}$ sites, e.g., $i=1, \ldots, N_{A}$, out of the total $N$ sites. Having obtained the path integral, we now seek to evaluate the integral at the large- $N$ saddle point, i.e., $N \rightarrow \infty$. This limit can be accessed by taking the disorder average over $J_{i_{1} \ldots i_{q} ; j_{q} \ldots j_{1}}$ s and then introducing the large- $N$ field

$$
G_{\sigma^{\prime} b, \sigma a}\left(\tau_{2}, \tau_{1}\right)=\frac{1}{N} \sum_{i} \bar{c}_{i \sigma a}\left(\tau_{1}\right) c_{i \sigma^{\prime} b}\left(\tau_{2}\right)
$$

and self-energy $\Sigma_{\sigma^{\prime} b, \sigma a}\left(\tau_{2}, \tau_{1}\right)$ for the fermions. We point out that the above analysis closely follows that of the thermalfield theory of the SYK model. The latter can be found in Refs. $[30,33,43,48,49]$, to name a few. The end result of introducing large- $N$ fields is an integral of the form

$$
\overline{\operatorname{Tr}\left[Z_{A}^{2}\right]^{r}}=\int \mathcal{D}(\bar{c}, c, \Sigma, G) d^{2}(\boldsymbol{\xi}, \boldsymbol{\eta}) e^{-\mathcal{S}[\bar{c}, c, \xi, \eta, \Sigma, G]},
$$

where the effective action $\mathcal{S}$ for the second Rényi entropy is bilinear in the Grassmann fields $\bar{c}, c, \xi$, and $\eta$, i.e.,

$$
\begin{aligned}
& \mathcal{S}=\left(-\sum_{i \in A, a=1}^{r}\left[\begin{array}{ll}
\bar{\xi}_{i a} & \bar{\eta}_{i a}
\end{array}\right]\left[\begin{array}{cc}
\frac{1}{2} & -\frac{1}{2} \\
\frac{1}{2} & \frac{1}{2}
\end{array}\right]\left[\begin{array}{l}
\xi_{i a} \\
\eta_{i a}
\end{array}\right]\right. \\
& +\sum_{i \in A, a=1}^{r} \int_{0}^{\beta} d \tau \delta\left(\tau^{+}\right)\left[\bar{c}_{i 1 a}(\tau) \quad \bar{c}_{i 2 a}(\tau)\right]\left[\begin{array}{c}
\xi_{i a} \\
\eta_{i a}
\end{array}\right] \\
& \left.-\sum_{i \in A, a=1}^{r} \int_{0}^{\beta} d \tau \delta(\tau)\left[\begin{array}{ll}
\bar{\xi}_{i a} & \bar{\eta}_{i a}
\end{array}\right]\left[\begin{array}{l}
c_{i 1 a}(\tau) \\
c_{i 2 a}(\tau)
\end{array}\right]\right) \\
& +\cdots \text {. }
\end{aligned}
$$

The Gaussian structure allows us to integrate the $\xi$ and $\eta$ variables, changing the effective action to another bilinear of $\bar{c}$ and $c$,

$$
\frac{1}{2^{N_{A} r}} \exp (-\int \sum_{i \in A, a} \overline{\boldsymbol{c}}_{i a}^{T}\left(\tau_{1}\right) \underbrace{\left[\begin{array}{rr}
1 & 1 \\
-1 & 1
\end{array}\right] \delta\left(\tau_{1}^{+}\right) \delta\left(\tau_{2}\right)}_{\boldsymbol{M}_{\sigma_{1} \sigma_{2}}\left(\tau_{1}, \tau_{2}\right)} \boldsymbol{c}_{\boldsymbol{i a}}\left(\tau_{2}\right)),
$$

where the matrix $M$ is defined as

$$
\boldsymbol{M}_{\sigma_{1} \sigma_{2}}\left(\tau_{1}, \tau_{2}\right)=\left[\begin{array}{rr}
1 & 1 \\
-1 & 1
\end{array}\right] \delta\left(\tau_{1}^{+}\right) \delta\left(\tau_{2}\right),
$$

and $c_{i a}(\tau)=\left[c_{i 1 a}(\tau) c_{i 2 a}(\tau)\right]^{T}$. Interestingly, at this point we see explicitly, from Eq. (63), how the fields $\xi, \eta$ extract the information regarding entanglement. Instead, of introducing complicated imaginary-time boundary conditions for fermions in subregion $A$, the fields alter the self-energy by providing a time-dependent "kick" to the fermions, while leaving the fermions outside $A$ untouched. All the fermions can now be integrated to produce $\overline{\operatorname{Tr}\left[Z_{A}^{2}\right]^{r}}=\int \mathcal{D}(\bar{c}, c, \Sigma, G) e^{-N \mathcal{S}[\Sigma, G]}$ and the final effective action

$$
\begin{aligned}
\mathcal{S}= & -(1-p) \ln \operatorname{det}\left(\boldsymbol{\partial}_{\boldsymbol{\tau}}+\boldsymbol{\Sigma}\right)-p \ln \operatorname{det}\left(\boldsymbol{\partial}_{\boldsymbol{\tau}}+\boldsymbol{\Sigma}+M\right) \\
& +\mathcal{S}_{G} \\
\mathcal{S}_{G}= & -\sum_{a b}^{r} \int d \tau_{1,2}(-1)^{q} \frac{J^{2}}{2 q} G_{\sigma^{\prime} b, \sigma a}^{q}\left(\tau_{2}, \tau_{1}\right) G_{\sigma a, \sigma^{\prime} b}^{q}\left(\tau_{1}, \tau_{2}\right) \\
& -\sum_{a b}^{r} \int d \tau_{1,2} \Sigma_{\sigma a, \sigma^{\prime} b}\left(\tau_{1}, \tau_{2}\right) G_{\sigma^{\prime} a, \sigma b}\left(\tau_{2}, \tau_{1}\right),
\end{aligned}
$$

which depends only on the Green's function $G$ and self-energy $\Sigma$. Here $\int d \tau_{1,2} \equiv \int_{0}^{\beta} d \tau_{1} d \tau_{2}$. The symbol $p$ denotes the the ratio of subsystem size to total size, i.e.,

$$
p=N_{A} / N,
$$


and can take a value from 0 to 1 . The symbols $\partial_{\tau}, \boldsymbol{\Sigma}$, appearing above, represents matrices having elements $\partial_{\tau_{1}} \delta\left(\tau_{1}-\right.$ $\left.\tau_{2}\right) \delta_{\sigma \sigma^{\prime}} \delta_{a b}, \Sigma_{\sigma a, \sigma^{\prime} b}\left(\tau_{1}, \tau_{2}\right)$ respectively. We evaluate the action $\mathcal{S}$ at the (disorder) replica diagonal and replica symmetric saddle point (i.e., $\Sigma, G \propto \delta_{a b}$ ) by minimizing with respect to $G$ and $\Sigma$ to get

$$
\begin{aligned}
\boldsymbol{G} & =(1-p) \tilde{\boldsymbol{G}}+p \boldsymbol{g}, \\
\tilde{\boldsymbol{G}} & =-\left(\boldsymbol{\partial}_{\boldsymbol{\tau}}+\boldsymbol{\Sigma}\right)^{-1}, \\
\boldsymbol{g} & =-\left(\boldsymbol{\partial}_{\boldsymbol{\tau}}+\boldsymbol{\Sigma}+\boldsymbol{M}\right)^{-1}, \\
\Sigma_{\sigma \sigma^{\prime}}\left(\tau_{1}, \tau_{2}\right) & =(-1)^{q+1} \boldsymbol{J}^{2} G_{\sigma \sigma^{\prime}}\left(\tau_{1}, \tau_{2}\right)^{q} G_{\sigma^{\prime} \sigma}\left(\tau_{2}, \tau_{1}\right)^{q-1},
\end{aligned}
$$

as the saddle-point conditions. Here $\boldsymbol{G}$ is the matrix representation of the Green's function $\boldsymbol{G}$ and $\boldsymbol{g}, \tilde{\boldsymbol{G}}$ are additional matrices that we have introduced to simplify the notation. Due to the similarities of the entanglement action $\mathcal{S}$ in Eq. (65) with the thermal free energy, we define the entanglement free-energy

$$
\begin{aligned}
& F_{\mathrm{EE}}(p, \beta) \\
& =\frac{1}{2 \beta} \mathcal{S} \\
& =\frac{1}{2 \beta}[p \ln \operatorname{det}(-\mathbf{g})+(1-p) \ln \operatorname{det}(-\tilde{\mathbf{G}}) \\
& \quad-\int_{0}^{\beta} d \tau_{1,2} \sum_{\sigma=1,2}(-1)^{q} \frac{J^{2}}{2 q} G_{\sigma^{\prime} \sigma}\left(\tau_{2}, \tau_{1}\right)^{q} G_{\sigma \sigma^{\prime}}\left(\tau_{1}, \tau_{2}\right)^{q} \\
& \left.\quad-\int_{0}^{\beta} d \tau_{1,2} \sum_{\sigma=1,2} \Sigma_{\sigma \sigma^{\prime}}\left(\tau_{1}, \tau_{2}\right) G_{\sigma^{\prime} \sigma}\left(\tau_{2}, \tau_{1}\right)\right]
\end{aligned}
$$

which allows us to express the second Rényi entropy [Eq. (56)] as

$$
S^{(2)}(p, \beta)=2 \beta\left[F_{\mathrm{EE}}(p, \beta)-F_{\mathrm{EE}}(p=0, \beta)\right],
$$

which depends on $p$ and temperature $\beta^{-1}$. Note that we have used the fact that thermal free energy $F(\beta)$ at the saddle point is given by $-\beta^{-1} \overline{\ln Z}$ and is equal to $F_{\mathrm{EE}}(p=0, \beta)$ in Eq. (69). The ground-state Rényi entanglement entropy for an arbitrary subsystem size $p$ can now be calculated by taking the limit

$$
S^{(2)}(p)=\lim _{\beta \rightarrow \infty} S^{(2)}(p, \beta) .
$$

The above analysis can also be carried out for the generalizations of the SYK model given in Eqs. (53), (55), and (54). The equations to evaluate the Rényi entropy for the strongly interacting Fermi liquid Hamiltonian in Eq. (53) and BA Hamiltonian in Eq. (54) can be similarly obtained and are given in Appendix E.

For extended systems of the kind described by Eq. (55), i.e., a ring of SYK dots connected to their nearest neighbors, we choose the subsystem as the $l$ successive dots, e.g., $x=$ $1, \ldots, l$ [see Fig. 2(d)] as the subsystem $A$. The saddle-point conditions for this arrangement are

$$
\left(\partial_{\tau}+\boldsymbol{\Sigma}^{(x)}+M \delta_{x \in A}\right) \boldsymbol{G}^{(x)}=-1,
$$

$$
\begin{aligned}
\Sigma_{\sigma \sigma^{\prime}}^{(x)}\left(\tau_{1}, \tau_{2}\right)= & (-1)^{q+1} J^{2} G_{\sigma \sigma^{\prime}}^{(x)}\left(\tau_{1}, \tau_{2}\right)^{q} G_{\sigma \sigma^{\prime}}^{(x)}\left(\tau_{2}, \tau_{1}\right)^{q-1} \\
& (-1)^{q_{\mathrm{hop}}+1} t_{\text {chain }}^{2}\left[G_{\sigma \sigma^{\prime}}^{(x-1)}\left(\tau_{1}, \tau_{2}\right)^{q_{\mathrm{hop}}}\right. \\
& \left.+G_{\sigma \sigma^{\prime}}^{(x+1)}\left(\tau_{1}, \tau_{2}\right)^{q_{\mathrm{hop}}}\right] G_{\sigma^{\prime} \sigma}^{(x)}\left(\tau_{2}, \tau_{1}\right)^{q_{\mathrm{hop}}-1},
\end{aligned}
$$

where the matrix $M$ [defined in Eq. (64)] adds to the sitedependent self-energy $-\Sigma^{(x)}$ only for dots which belong to subsystem $A$, as indicated by the Kronecker $\delta$ function $\delta_{x \in A}$. The Green's function $G^{(x)}$ also become dependent on the site index $x$. The entanglement free energy can be calculated in terms of the space-dependent $G^{(x)}$ and $\Sigma^{(x)}$, i.e.,

$$
\begin{aligned}
F_{\mathrm{EE}}(p, \beta) & \\
= & \frac{1}{2 \beta}\left[\sum_{x} \ln \operatorname{det}\left(-\mathbf{G}^{(x)}\right)\right. \\
& -\int_{0}^{\beta} d \tau_{1,2}\left\{\sum_{x, \sigma}(-1)^{q} \frac{J^{2}}{2 q} G_{\sigma^{\prime} \sigma}^{(x)}\left(\tau_{2}, \tau_{1}\right)^{q} G_{\sigma \sigma^{\prime}}^{(x)}\left(\tau_{1}, \tau_{2}\right)^{q}\right. \\
& +\sum_{x, \sigma} \Sigma_{\sigma \sigma^{\prime}}^{(x)}\left(\tau_{1}, \tau_{2}\right) G_{\sigma^{\prime} \sigma}^{(x)}\left(\tau_{2}, \tau_{1}\right) \\
& \left.\left.+\sum_{\left\langle x x^{\prime}\right\rangle \sigma \sigma^{\prime}} \frac{(-1)^{q_{\mathrm{hop}}} t_{\text {chain }}^{2}}{q_{\text {hop }}} G_{\sigma \sigma^{\prime}}^{(x)}\left(\tau_{1}, \tau_{2}\right)^{q_{\mathrm{hop}}} G_{\sigma^{\prime} \sigma}^{\left(x^{\prime}\right)}\left(\tau_{2}, \tau_{1}\right)^{q_{\mathrm{hop}}}\right\}\right],
\end{aligned}
$$

where $\left\langle x x^{\prime}\right\rangle$ represents nearest neighbors. The presence of $\delta_{x \in A}$ in Eq. (71) explicitly breaks translation symmetry and one needs to retain the Green's functions for each site $x$ in order to calculate the entanglement entropy.

We obtain the Rényi entropy in Sec. III for all the models discussed in this section by numerically solving the saddlepoint equations, such as Eqs. (67) and (71), and using Eq. (69). We discuss the iterative numerical algorithm to solve the saddle-point equations in Appendix $\mathrm{H} 2$.

\section{E. Rényi entropy in Hubbard model: Dynamical mean field theory (DMFT)}

In this section, we demonstrate the application of the pathintegral method of Sec. II B for the Hubbard model. We develop the formulation to compute Rényi entropy within the dynamical mean field theory (DMFT) [29], one of the successful approaches to treat electronic correlation and metal-insulator transition in Hubbard model. We formulate the DMFT for Rényi entropy in the single-site approximation. In the usual single-site DMFT [29], one reduces a lattice problem into an impurity coupled to a bath and the properties of the impurity and the bath are calculated self-consistently using the noninteracting dispersion. We extend the DMFT approximation to evaluate the path integrals developed in Sec. II B. The method can be integrated with the continuous-time quantum Monte Carlo (CTQMC) impurity solver [26] and extended to the cluster implementations [68]. Here we only discuss the formulation of the problem within DMFT; the numerical implementation will be discussed in a future publication [69].

We consider the Hubbard model

$$
H=\sum_{(i j), \sigma} t_{i j} c_{i \sigma}^{\dagger} c_{j \sigma}-\mu \sum_{i} n_{i}+U \sum_{i} n_{i \uparrow} n_{i \downarrow}
$$


with hopping amplitudes $t_{i j}$ between sites on a lattice, $\mu$ the chemical potential, and $U$ the on-site repulsive interaction between fermions with opposite spins $\sigma=\uparrow, \downarrow$. Here $n_{i \sigma}=$ $c_{i \sigma}^{\dagger} c_{i \sigma}$ and $n_{i}=\sum_{\sigma} n_{i \sigma}$ are the electronic number operators.

We discuss the second Rényi entropy of a subsystem for equilibrium state described by the thermal density matrix [Eq. (13)]. The method can be easily extended to a nonequilibrium situation (Sec. II B) via nonequilibrium DMFT [70]. As in Eq. (56), we compute

$$
S^{(2)}=\beta\left(\Omega^{(2)}-2 \Omega\right),
$$

where $\Omega^{(2)}=-T \ln Z^{(2)}\left(Z^{(2)} \equiv \operatorname{Tr}_{A} Z_{A}^{2}\right)$ and $\Omega=-T \ln Z$ are relevant grand potentials. Again, using the trace identity [Eq. (57)], we obtain the path integral

$$
\begin{aligned}
Z^{(2)}= & \int \prod_{i \in A, \sigma, \alpha=1,2} d^{2} \xi_{i \sigma \alpha} f_{N}(\xi) \int \mathcal{D}(\bar{c}, c) e^{-\mathcal{S}}, \\
\mathcal{S}= & \int_{0}^{\beta} d \tau\left[\sum_{i \sigma \alpha} \bar{c}_{i \sigma \alpha}\left[\left(\partial_{\tau}-\mu\right) \delta_{i j}+t_{i j}\right] c_{j \sigma \alpha}\right. \\
& \left.+U \sum_{i \alpha} n_{i \uparrow \alpha} n_{i \downarrow \alpha}\right] \\
& +\sum_{i \in A, \sigma \alpha} \int_{0}^{\beta} d \tau\left[\bar{\xi}_{i \sigma \alpha} \delta(\tau) c_{i \sigma \alpha}(\tau)-\delta\left(\tau^{+}\right) \bar{c}_{i \sigma \alpha}(\tau) \xi_{i \sigma \alpha}\right] .
\end{aligned}
$$

Here we have the auxiliary Grassmann fields $\xi=$ $\left\{\bar{\xi}_{i \sigma \alpha}, \xi_{i \sigma \alpha}\right\}_{i \in A}$ with spin $(\sigma)$ and entanglement replica $(\alpha=$ $1,2)$ indices, and $f_{N}(\xi)=\exp \left[-(1 / 2) \sum_{i \in A, \sigma}\left(\bar{\xi}_{i \sigma 1} \xi_{i \sigma 1}+\right.\right.$ $\left.\left.\bar{\xi}_{i \sigma 2} \xi_{i \sigma 2}-\bar{\xi}_{i \sigma 1} \xi_{i \sigma 2}+\bar{\xi}_{i \sigma 2} \xi_{i \sigma 1}\right)\right]$. As in the case of chain of SYK dots in Sec. II D 5, due to the entanglement subdivision, the above action breaks translational invariance. Hence, we use a single-site inhomogeneous DMFT to reduce the lattice problem [Eq. (73)] into an impurity problem for site $i$,

$$
\begin{aligned}
Z_{i}^{(2)}= & \int\left(d^{2} \boldsymbol{\xi} f_{N}(\xi)\right)^{\delta_{i \in A}} \mathcal{D}(\bar{c}, c) e^{-\mathcal{S}_{i}}, \\
\mathcal{S}_{i}= & -\int d \tau_{1,2} \sum_{\sigma \alpha \gamma} \bar{c}_{\sigma \alpha}\left(\tau_{1}\right) \mathcal{G}_{i \alpha \gamma}^{-1}\left(\tau_{1}, \tau_{2}\right) c_{\sigma \gamma}\left(\tau_{2}\right) \\
& +U \int_{0}^{\beta} d \tau \sum_{\alpha} n_{\uparrow \alpha}(\tau) n_{\downarrow \alpha}(\tau) \\
& +\delta_{i \in A} \sum_{\sigma \alpha} \int_{0}^{\beta} d \tau\left[\bar{\xi}_{\sigma \alpha} \delta(\tau) c_{\sigma \alpha}(\tau)-\delta\left(\tau^{+}\right) \overline{\boldsymbol{c}}_{\sigma \alpha}(\tau) \xi_{\sigma \alpha}\right] .
\end{aligned}
$$

The auxiliary Grassmann fields $\left\{\bar{\xi}_{\sigma \alpha}, \xi_{\sigma \alpha}\right\}$ only appears for sites belonging to the $A$ region; $d^{2} \xi=\prod_{\underline{\sigma} \alpha} d^{2} \xi_{\sigma \alpha}$ and $\quad f_{N}(\xi)=\exp \left[-(1 / 2) \sum_{\sigma}\left(\bar{\xi}_{\sigma 1} \xi_{\sigma 1}+\bar{\xi}_{\sigma 2} \xi_{\sigma 2}-\bar{\xi}_{\sigma 1} \xi_{\sigma 2}+\right.\right.$ $\left.\left.\bar{\xi}_{\sigma 2} \xi_{\sigma 1}\right)\right]$. The above impurity action could be derived from Eq. (75) using the cavity method [29]. To simplify the notations, we have assumed a paramagnetic state with spin symmetry, and hence the dynamical Weiss mean field is independent of spin and is given by

$$
\mathcal{G}_{i}^{-1}\left(\tau, \tau^{\prime}\right)=-\left(\partial_{\tau}-\mu\right) \delta\left(\tau-\tau^{\prime}\right) \mathcal{I}-\Delta_{i}\left(\tau, \tau^{\prime}\right),
$$

a $2 \times 2$ matrix in the replica space, where $\mathcal{I}$ denotes a $(2 \times 2)$ unit matrix and $\Delta_{i}\left(\tau, \tau^{\prime}\right)$ is the hybridization function which carries the information about the lattice and needs to be found self-consistently (see below). For $i \in A$, we integrate out the entangling Grassmann fields $\bar{\xi}_{\sigma \alpha}, \xi_{\sigma \alpha}$ to get $Z_{i}^{(2)}=$ $\int \mathcal{D}(\bar{c}, c) e^{-\tilde{\mathcal{S}}_{i}}$ and the effective action

$$
\begin{gathered}
\tilde{\mathcal{S}}_{i}=-\int d \tau_{1,2} \sum_{\sigma \alpha \gamma} \bar{c}_{\sigma \alpha}\left(\tau_{1}\right) \tilde{\mathcal{G}}_{i \alpha \gamma}^{-1}\left(\tau_{1}, \tau_{2}\right) c_{\sigma \gamma}\left(\tau_{2}\right) \\
+U \int_{0}^{\beta} d \tau \sum_{\alpha} n_{\uparrow \alpha}(\tau) n_{\downarrow \alpha}(\tau), \\
\tilde{\mathcal{G}}_{i}^{-1}\left(\tau, \tau^{\prime}\right)=-\left(\partial_{\tau}-\mu\right) \delta\left(\tau-\tau^{\prime}\right) \mathcal{I}-\Delta_{i}\left(\tau, \tau^{\prime}\right) \\
-\delta_{i \in A} M\left(\tau, \tau^{\prime}\right),
\end{gathered}
$$

where the $2 \times 2$ matrix $M$ is given in Eq. (64). The impurity Green's function $G_{i}\left(\tau, \tau^{\prime}\right)$ can be obtained from the impurity Dyson equation,

$$
G_{i}^{-1}\left(\tau, \tau^{\prime}\right)=\tilde{\mathcal{G}}_{i}^{-1}\left(\tau, \tau^{\prime}\right)-\Sigma_{i}\left(\tau, \tau^{\prime}\right),
$$

where $\Sigma_{i, \alpha \gamma}\left(\tau, \tau^{\prime}\right)$ is the impurity self-energy that needs to be computed using an appropriate impurity solver, e.g., iterative perturbation theory (IPT) [29] or CTQMC [26]. The hybridization function can be obtained in terms of cavity Green's function as

$$
\Delta_{i \alpha \gamma}\left(\tau, \tau^{\prime}\right)=\sum_{j k} t_{i j} t_{i k} G_{j \alpha, k \gamma}^{(i)}\left(\tau, \tau^{\prime}\right) .
$$

Here $G^{(i)}\left(\tau, \tau^{\prime}\right)$ is the Green's function with $i$ th site removed from the lattice. The cavity Green's function can be obtained from the lattice Green's functions as

$$
\begin{aligned}
G_{j \alpha, k \gamma}^{(i)}\left(\tau, \tau^{\prime}\right)= & G_{j \alpha, k \gamma}\left(\tau, \tau^{\prime}\right)-\sum_{\eta \delta} \int d \tau_{1,2}\left[G_{j \alpha, i \eta}\left(\tau, \tau_{1}\right)\right. \\
& \left.\times G_{i \eta, i \delta}^{-1}\left(\tau_{1}, \tau_{2}\right) G_{i \delta, k \gamma}\left(\tau_{2}, \tau^{\prime}\right)\right]
\end{aligned}
$$

where $G\left(\tau, \tau^{\prime}\right)$ is the full lattice Green's function. To obtain the full lattice Green's function, within the single-site DMFT approximation, one assumes that the lattice self-energy is local and is given by the impurity self-energy. Then, the lattice Green's function is obtained from the Dyson equation

$$
\begin{aligned}
& \sum_{j} \int d \tau_{1}\left[-\left\{\left(\partial_{\tau}-\mu\right) \delta_{i j}+t_{i j}\right\} \delta\left(\tau-\tau_{1}\right) \mathcal{I}\right. \\
& \left.\quad+\delta_{i \in A} \delta_{i j} M\left(\tau, \tau_{1}\right)-\delta_{i j} \Sigma_{i}\left(\tau, \tau_{1}\right)\right] G_{j k}\left(\tau, \tau^{\prime}\right) \\
& =\delta_{i k} \delta\left(\tau-\tau^{\prime}\right) \mathcal{I} .
\end{aligned}
$$

The above equation can be used to obtain the hybridization function in Eq. (82) and thus closes the DMFT selfconsistency loop. The numerical solution of the above DMFT equations is rather involved since both space and time translation invariance are broken, the former due to the choice of subregion $A$ and the latter due to the time-dependent selfenergy kick used to extract the entanglement. The numerical solution will be discussed in a future work [69].

We conclude this section by briefly mentioning the important step to compute the grand potentials $\Omega^{(2)}$ and $\Omega$ appearing in Eq. (74) for the second Rényi entropy. The grand potentials can be obtained via coupling constant integration discussed in 
detail in Appendix F. In this method, one considers a modified Hamiltonian $H_{\lambda}=H_{0}+\lambda H_{1}$, where $H_{0}$ is the noninteracting part in Eq. (73) and $H_{1}$ is the Hubbard term with the coupling constant $0 \leqslant \lambda \leqslant 1 ; \lambda=0$ is the noninteracting limit and the $\lambda=1$ is the interacting Hamiltonian of interest. The grand potentials could be obtained as

$$
\begin{aligned}
\Omega^{(2)}= & \Omega^{(2)}(0)+\int_{0}^{1} \frac{d \lambda}{\lambda} \sum_{i j \sigma}\left[\left(\partial_{\tau_{0}}-\mu\right) \delta_{i j}+t_{i j}\right] \\
& \times G_{j \sigma \alpha, i \sigma \alpha}^{(\lambda)}\left(0,0^{+}\right), \\
\Omega= & \Omega(0)+\int_{0}^{1} \frac{d \lambda}{\lambda} \sum_{i j \sigma}\left[\left(\partial_{\tau}-\mu+\epsilon_{i}\right) \delta_{i j}+t_{i j}\right] \\
& \times G_{j \sigma, i \sigma}^{(\lambda)}\left(\tau, \tau^{+}\right) .
\end{aligned}
$$

The integrands above can be calculated by computing the Green's function $G^{(\lambda)}$ via DMFT for each $\lambda$. One important point here is that the equal-time Green's function appearing in the expression for $\Omega^{(2)}$ needs to be calculated at the imaginary-time instant where the fermionic source fields are inserted in the path integral, in our case $\tau=0$. In contrast, $\tau$ in the expression of usual thermodynamic grand potential $\Omega$ is arbitrary. $\Omega^{(2)}(0)$ and $\Omega(0)$ are the grand potentials for the noninteracting system $(\lambda=0)$ which can be calculated directly using the results of Sec. II C.

\section{ANALYTICAL AND NUMERICAL RESULTS FOR RÉNYI ENTROPY IN LARGE- $N$ FERMIONIC MODELS OF FERMI LIQUIDS AND NON-FERMI LIQUIDS}

With the formalism for calculating Rényi entropy for large$N$ systems in place, we proceed forward and discuss the solutions obtained for the models introduced in Sec. II D. In this paper, we only describe the results for the second Rényi entropy obtained from the thermal field theory in Sec. II D 5. The results for higher order Rényi entropies and time evolution of Rényi entropy in nonequilibrium situations will be communicated in a future work [71].

\section{A. Noninteracting large- $N$ model with disorder}

To set the stage and benchmark our large- $N$ field theory formalism of Sec. II D 5, we first discuss the Rényi entropy in a noninteracting model. For $q=1$, the model defined in Eq. (52) describes a noninteracting system connected with disordered random hoppings having a variance $t_{\text {hop }}^{2}$, and hence is amenable to solution in multiple ways. Therefore, we compare the temperature-dependent Rényi entropy, defined in Eq. (69), calculated using (a) the correlation matrix approach applicable for noninteracting system as discussed in Sec. II C, where the correlation matrix is evaluated by diagonalizing the noninteracting Hamiltonian, (b) using many-body exact diagonalization (ED) (see Appendix H 1), and (c) using the large- $N$ thermal field developed in Sec. II D 5. For cases (a) and (b), the Rényi entropy is calculated by explicitly averaging over multiple disorder realizations.

Figure 3 shows the variation of the second Rényi entropy with subsystem size $p$ for multiple temperatures calculated using the above methods. We get an excellent match for

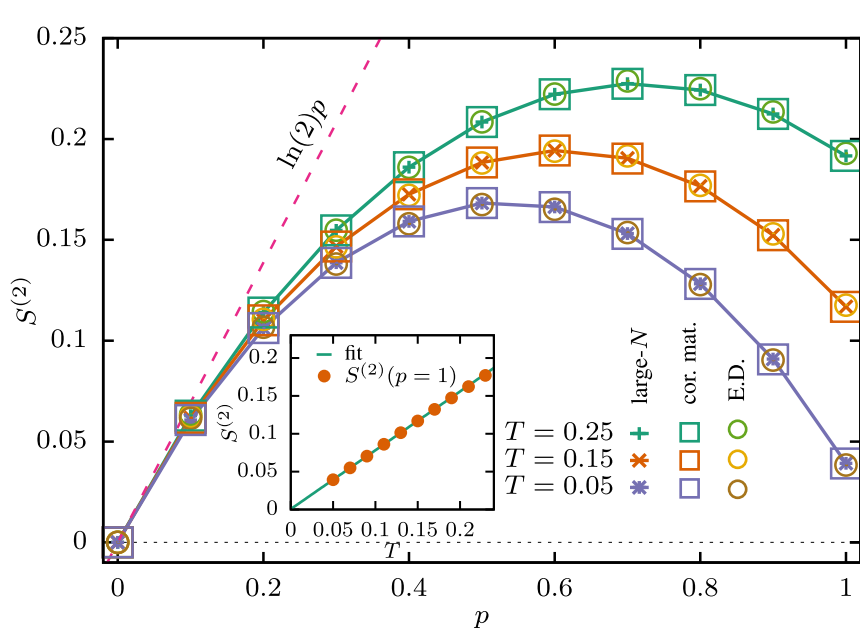

FIG. 3. Rényi entropy in noninteracting large- $N$ model: Second Rényi entropy, $S^{(2)}$, for the noninteracting model with random hoppings shown as a function of subsystem fraction $p$ for several values of temperature $T$. The predictions obtained from large- $N$ saddlepoint theory (points with lines) show an excellent agreement with results (represented by color matched squares) obtained using the correlation matrix, as well as exact diagonalization (ED) calculations (circles) performed for $N=10$ sites. All three techniques show a maximal entanglement scaling of Rényi entropy for small subsystem sizes $(p \rightarrow 0)$ with a $\ln (2)$ coefficient. Inset gives the temperature dependence of the Rényi entropy at $p=1$, i.e., $S^{(2)}(p=1)$, which goes to zero as temperature is decreased, indicating that the thermal density matrix, used for calculating entanglement, approaches the ground state for the model.

all three cases. Particularly remarkable is the fact that the large- $N$ results almost exactly match with those obtained with ED for quite small system size $(N=10)$, implying that $1 / N$ corrections to entanglement entropy are rather small. This feature persists for the interacting large- $N$ models discussed in the next sections. We find that for small subsystem size, i.e., $p \rightarrow 0$, the Rényi entropy grows with $p$ with a slope of $\ln (2)$ (see dashed line in Fig. 3), indicating a maximal entanglement which can be argued based on the counting of degrees of freedom [63,72]. In the limit of large subsystem size, i.e., $p \rightarrow 1$, the Rényi entropy attains a finite nonzero value that goes to zero as $T \rightarrow 0$; see Fig. 3 inset. At finite temperature, the Rényi entropy of the subsystem for a thermal mixed-state density matrix picks up contributions from the usual thermal entropy, which is only of statistical origin. However, as temperature is lowered $(\beta \rightarrow \infty)$, the thermal density matrix approaches that of the ground state, i.e., $\rho^{2} \rightarrow \rho$. For any pure state, in this case the ground state, the entanglement entropy goes to zero as the subsystem size approaches the size of the parent system. This, however, is not the case for the SYK model in the large- $N$ limit, as discussed below.

\section{B. SYK model: Rényi entropy of a non-Fermi liquid}

We now move on to large- $N$ interacting Hamiltonians, starting with the original $q=2$ SYK model described in Eq. (52). The model connects fermions, on $N$ sites, via allto-all two-body interactions with random complex amplitudes having variance $J^{2}$, which we set to unity for this section. 
There have been several numerical and analytical works on entanglement entropy [43,63-66,72] in the SYK model for various different contexts. The analytical approaches have used standard replica path-integral approach for Rényi entropy. Among these works, Gu et al. have studied growth of bipartite Rényi entanglement entropy in a chain of SYK dots starting from a thermofield double state [43]. They made a diagonal approximation in the entanglement replica space for weak interdot coupling. The diagonal approximation was justified based on the argument that the replica off-diagonal terms were perturbatively small in the interdot coupling. In our work, we find the (entanglement) replica off-diagonal terms to be very important for the Rényi entropy for a subsystem within a single dot, since the intradot coupling, of course, cannot be treated perturbatively. In Sec. III E, for the higher dimensional models, we consider strong interdot coupling and replica off-diagonal terms are also substantial there. Reference [65] has used an approximation similar to that in Ref. [43] to look into ground-state entanglement between two quadratically coupled SYK dots. During the preparation of our paper, we became aware of a very recent work [66] on subsystem Rényi entropy in the thermal ensemble for the SYK model using a standard approach with the boundary conditions. Overall, their results compares well with our results on SYK model obtained using the field-theoretic method developed in this work.

As in the noninteracting large- $N$ model case, we compare the Rényi entropy obtained via large- $N$ thermal field theory with that obtained via ED. However, the Rényi entropy cannot be calculated in the interacting case using the correlation matrix approach. Figure 4(a) gives the second Rényi entropy with subsystem size $p$ for several values of $T$. We find that, yet again, for all temperature values the results from large- $N$ and ED calculations agree really well, when $p<0.4$, even for exact diagonalization performed for $N=12$ sites. Also as expected, volume law with a $\ln (2)$ coefficient still holds as $p \rightarrow 0$ for all temperature values. Interestingly though, as $T \rightarrow 0$, the large- $N$ result deviates substantially from the ED result when $p \geqslant 0.4$ and especially when $p \rightarrow 1$. While the Rényi entanglement entropy for the ground state, obtained by $\mathrm{ED}$, goes to zero as expected, the large- $N$ result seems to converge linearly to a finite value when $T$ is reduced as shown in Fig. 4(b), where we plot $S^{(2)}(p=1)$ for low values of $T$. In fact, when extrapolated to $T \rightarrow 0$, we find the intercept to be equal (within numerical accuracy) to the thermal residual entropy $S_{0} \approx 0.464 \ldots$ of the SYK model. Remarkably, the residual entropy has found a way to influence the zerotemperature Rényi entropy of the SYK model in the large- $N$ limit. An explanation is provided when we look back at the expression for Rényi entropy in Eq. (69). Since by definition $\lim _{p \rightarrow 1} F_{\mathrm{EE}}(p, \beta)=F(2 \beta)$, expanding the entanglement free energy to first order in $T\left(=\beta^{-1}\right)$ we find

$$
\begin{aligned}
S^{(2)}(p \rightarrow 1) & =\lim _{T \rightarrow 0} \frac{F(T / 2)-F(T)}{T / 2}=\lim _{T \rightarrow 0} \frac{\frac{T}{2} F(0)-T F(0)}{T / 2} \\
& =-\left.\frac{\partial F}{\partial T}\right|_{T=0}=S_{0},
\end{aligned}
$$

i.e., $S^{(2)}(p=1)$ is indeed equal to the residual entropy $S_{0}$. The above analysis suggests that the limit $N \rightarrow \infty$ and $T \rightarrow 0$ do
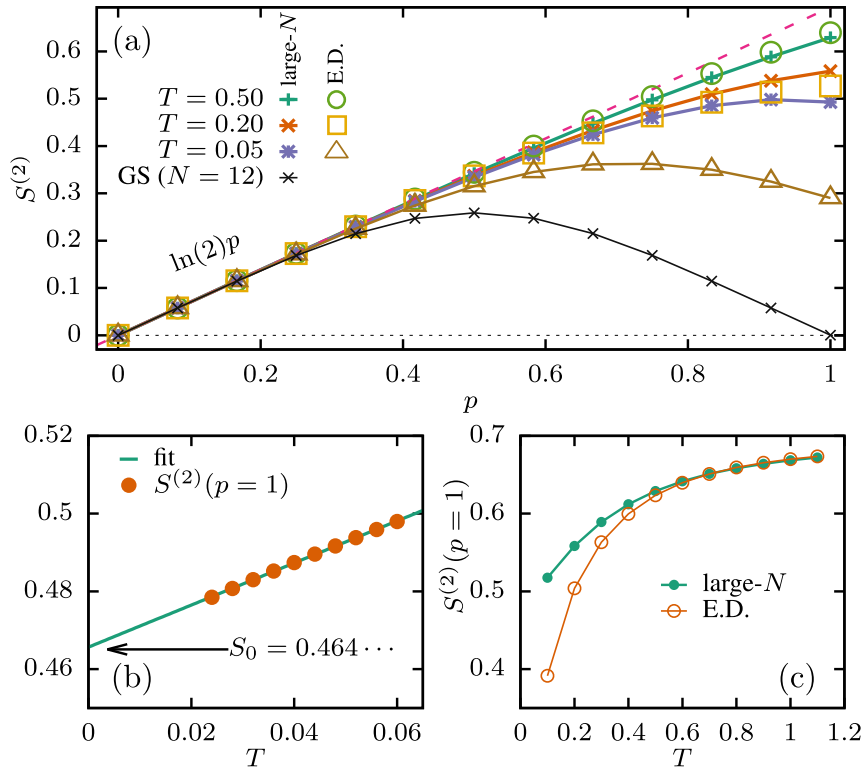

FIG. 4. Rényi entropy in SYK model: (a) The second-Rényi entropy $\left(S^{(2)}\right)$ for the SYK $(q=2)$ model as a function of subsystem size $p$ for temperature values $T=0.50,0.20,0.05$. Predictions from large- $N$ formalism (points with lines) match exactly with ED calculations (represented by circles, squares, and triangles) for subsystem sizes $p<0.4$ [both showing a volume-law scaling with a $\ln (2)$ coefficient], but deviate significantly at larger $p$ values due to the influence of residual-thermal entropy $S_{0}$ of the SYK model. The $S^{(2)}$ vs $p$ curve (black line with crosses) for the ground-state of the model, obtained using ED, is also shown for comparison. (b) Rényi entropy for $p=1$, i.e., $S^{(2)}(p=1)$, approaches $S_{0}$ linearly when temperature $T$ goes to zero, as seen explicitly from a linear fit (line) to the large$N$ data (circles) and recovering an intercept equal to $S_{0} \approx 0.464$. (c) The match between large- $N$ prediction (filled circles) and $\mathrm{ED}$ (empty circles), for $S^{(2)}(p=1)$, is recovered at higher temperatures; see text for explanation. All ED calculations were done for $N=12$ sites.

not commute. It is established that the residual entropy for the SYK model is a consequence of exponentially small in $N$ level spacings arising from the large- $N$ limit. Taking the large- $N$ limit first also prohibits "direct" access to the ground-state quantum entanglement when temperature is reduced, since any $T$ however small cannot resolve these exponentially small level spacings. We therefore argue that temperatures higher than the typical level spacings obtained from ED should "mimic" this large- $N$ effect and predictions from large- $N$ thermal field theory should match with finite- $N$ ED calculations. To test this hypothesis, we plot the $p=1$ value for the second Rényi entropy obtained from large- $N$ and finite- $N$ ED calculations for higher values of temperature in Fig. 4(c). Indeed, we find that the results match perfectly for $T \geqslant 0.5$, thereby validating our intuition about the role of residual entropy in $T=0$ Rényi entropy at large $N$.

\section{SYK model with disordered hopping: Rényi entropy of an interacting Fermi liquid}

We now discuss the behavior of entanglement in an interacting Fermi liquid, like the one described by the Hamiltonian 

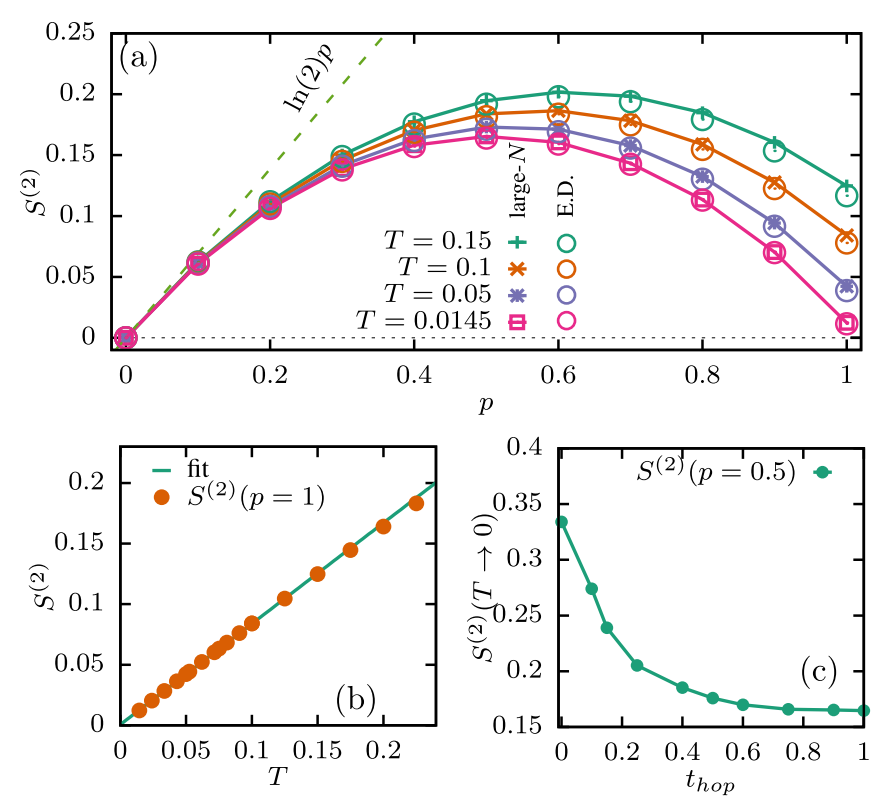

FIG. 5. Rényi entropy of interacting Fermi liquid: (a) Subsystem size $(p)$ dependence of second Rényi entropy, $S^{(2)}$, in the zerodimensional Fermi-liquid model [defined in Eq. (53)] with SYK interactions, for several values of temperature $T$. The strength of hopping $t_{\text {hop }}$ and interactions $J$ are both set to one. The predictions from the large- $N$ formalism (points) and results from exact diagonalization (circles) match for all values of $p$ and $T$. Both predict a volume-law scaling with a $\ln (2)$ coefficient. (b) The Rényi entropy for $p=1$ (represented by circles) shows a linear dependence on $T$ (for values less than $T=0.15$ ) and goes to zero as $T \rightarrow 0$, indicating an approach to a ground state with no residual-thermodynamic entropy. (c) The zero-temperature bipartite second Rényi entropy, $S^{(2)}(p=0.5, T \rightarrow 0)$, shown as a function of increasing hopping strength $t_{\text {hop }}(J=1)$, with $t_{\text {hop }}=0$ being the SYK-NFL limit. As hopping increases, bipartite Rényi entropy decrease from a rather large value $(\approx 0.35)$ and saturates to a value $(\approx 0.15)$ close to that of the noninteracting model (see Fig. 3).

in Eq. (53). The addition of a $q=1$ quadratic term $t_{i j} c_{i}^{\dagger} c_{j}$ has been shown $[30,72]$ to change the SYK NFL ground state to an interacting zero-dimensional Fermi liquid. We set $t_{\text {hop }}=1$, $J=1$ for the variances of $t_{i j}, J_{i j}$ in Eq. (53) and compare the second Rényi entropy obtained from large- $N$ theory with ED in Fig. 5(a). Quite encouragingly, we find an excellent match between the two for all values of temperatures. As expected, the volume law for entanglement is shown to hold as $p \rightarrow 0$ due to the all-to-all nature of interactions and hoppings. Interestingly, this time, unlike the SYK NFL case, the entanglement entropy for $p=1$ goes to zero as temperature approaches zero [see Fig. 5(b)]. Looking back at the analysis in Eq. $(87), S^{(2)}(p \rightarrow 1) \rightarrow 0$ implies that the FL ground state does not possess any residual entropy. Indeed, we find in literature [73] that the presence of a quadratic term in Eq. (53) drastically reduces the exponentially large density of levels near the ground state, thereby reducing the residual entropy to zero.

It is interesting to explore how entanglement entropy changes as the relative strength of interactions is increased with respect to the quadratic term, going from a noninteracting system to a NFL, via heavy Fermi liquid states for $t_{\text {hop }} \ll J$. To this end, we set $J=1$ and track the bipartite Rényi entropy, i.e., $S^{(2)}(p=1 / 2)$, as the value of $t_{\text {hop }}$ is increased from 0 to 1 . The bipartite Rényi entropy is the maximal value that can be attained for any homogeneous system. Figure 5(c) shows the result of this exercise. When $t_{\text {hop }}=0$, the model is described by purely SYK-type interactions and therefore bipartite Rényi entropy attains the maximum possible value $\approx 0.5 \ln (2)$. Interestingly, as $t$ is increased, the entanglement decreases continuously, indicating that a FL of this type becomes less entangled when the relative strength of interactions is reduced. Finally, for values of $t_{\text {hop }} \geqslant J$ the bipartite entanglement entropy saturates to around 0.16 , a value close to that of noninteracting large- $N$ model (see Fig. 3), implying that interactions become irrelevant in this limit.

\section{BA model: Rényi entropy across a non-Fermi liquid to Fermi liquid transition}

One of the important aspects of the putative duality between SYK model and black holes in quantum gravity [27,33$35]$ is the residual entropy and its possible connection with the black hole entropy. Hence, it is interesting to ascertain if there is any relation between the residual entropy and the ground-state entanglement entropy of SYK NFL. We saw that the residual entropy inevitably appears in the Rényi entropy when the large- $N$ limit is taken followed by the $T \rightarrow 0$ limit in the pure SYK model. Here we discuss the BA model [30], which helps us to make the connection between the residual and $T=0$ Rényi entropy more explicit by tuning a QPT between a SYK NFL and a FL.

The BA model is defined in Eq. (54). A $T=0$ transition between the chaotic NFL fixed point and nonchaotic FL fixed point is achieved by tuning the ratio of sites $p=N_{\psi} / N_{c}$. The quantum-critical point (QPT) occurs at $p=1$, below which the $c$ fermions behave as a SYK NFL and the whole system has a finite $p$-dependent residual entropy, $S_{0}(p)=[(1-$ $p) /(1+p)] S_{0}$, that goes to zero at the critical point [30]. Motivated by this, we ask whether any such sharp features exists in the Rényi entropy. We analyze the second Rényi entropy for the model at the large- $N$ saddle point (see Appendix E) using the following two choices of subsystem $A$ : (a) when $A$ is made up of all the $c$ fermions that have the SYK type interactions and (b) when the $A$ is composed of the all the noninteraction $\psi$ fermions. For our calculations, we set $J=t_{\text {hop }}=V=1$ [see Eq. (54)].

The subsystem-Rényi entropy for the $c$-fermions, $S_{c}^{(2)}$ [case (a)], and $\psi$ fermions, $S_{\psi}^{(2)}$ [case (b)], are shown as a function of site fraction $p$ in Fig. 6(a) for multiple values of temperature $T$. Ideally, at $T=0$, in a pure state one expects $S_{c}^{(2)}=S_{\psi}^{(2)}$. However, yet again, we find that residual entropy $S_{0}(p)$ has managed to influence entanglement entropy as seen from $S_{c}^{(2)}$ (represented by circles) approaching a value close to $S_{0}=0.464$ of the SYK model when $p \rightarrow 0$. On the other hand, the $S_{\psi}^{(2)}$ (triangles) goes to zero in the same limit in accordance with the volume-law maximal entanglement, $p \ln (2)$. To uncover the signature of the underlying QPT, we extrapolated $S_{c}^{(2)}$ and $S_{\psi}^{(2)}$ to $T \rightarrow 0$ and plot the results in Fig. 6(b). Remarkably, we find that around the critical point, $p=1$, the Rényi entropy for the fermions becomes exactly 

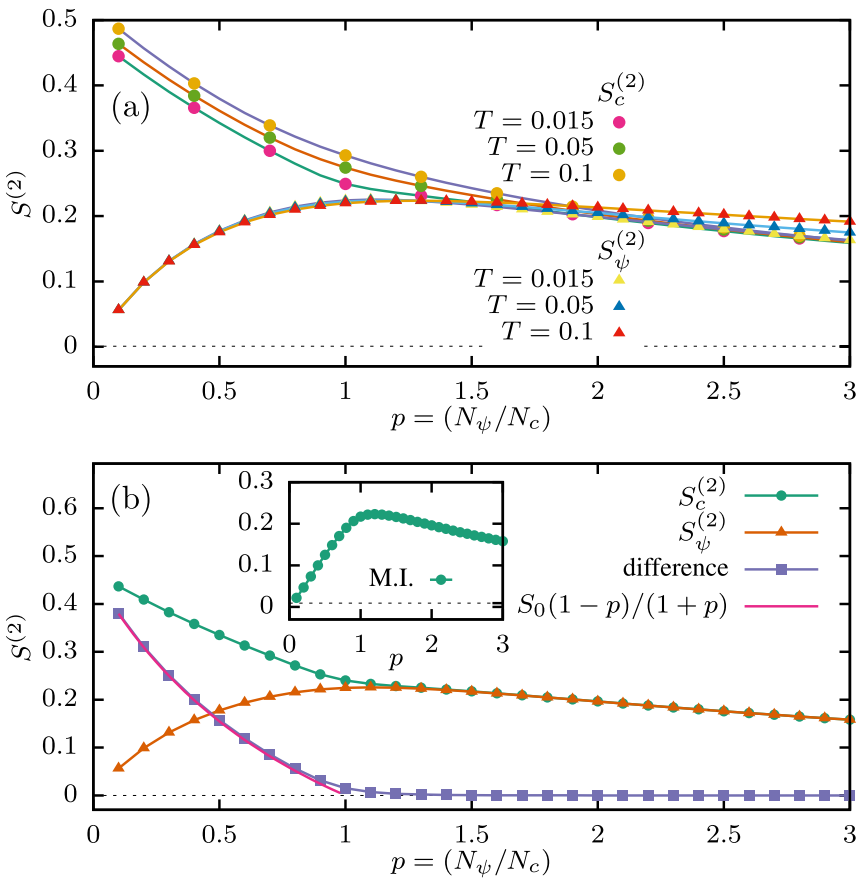

FIG. 6. Rényi entropy in BA model: (a) Subsystem second Rényi entropy for the $c$ fermions $S_{c}^{(2)}$ (circles with lines) and $\psi$ fermions $S_{\psi}^{(2)}$ (triangles), for the BA model [Eq. (54)], shown as function of site fraction $p=N_{\psi} / N_{c}$ for temperatures $T=0.015,0.05,0.1$. (b) Extrapolated zero-temperature Rényi entropies, $S_{c}^{(2)}$ (circles) and $S_{\psi}^{(2)}$ (triangles), showing the signature of the underlying QPT. $S_{c}^{(2)}$ and $S_{\psi}^{(2)}$ become exactly equal at the critical point for the QPT, $p=1$, and remain equal for $p>1$. Inset gives the mutual information (MI) between the $c$ and $\psi$ fermions showing a peak at the critical point. Furthermore, the difference of the Rényi entropies, $S_{c}^{(2)}-S_{\psi}^{(2)}$ (squares with lines), precisely matches the analytical prediction, $S_{0}(p)=S_{0}(1-p) /(1+p)$ (solid line), for the $p$-dependent residual entropy for the BA model from Ref. [30].

equal, i.e., $S_{c}^{(2)}=S_{\psi}^{(2)}$ and remains equal for all values $p>$ 1. Motivated by this, to further understand the relationship between the residual entropy $S_{0}(p)$ and the Rényi entropies $S_{c}^{(2)}, S_{\psi}^{(2)}$, we compare $S_{0}(p)$ with the difference $S_{c}^{(2)}-S_{\psi}^{(2)}$, which approaches $S_{0}$ for $p \rightarrow 0$ and also vanishes continuously at $p=1$. Quite encouragingly, we find the match to be excellent, as seen in Fig. 6(b). Thus, the difference between the Rényi entropies of the larger and smaller subsystems directly corresponds to the residual entropy and carries the signature of the underlying QPT. The preceding results also leads us to conjecture that the zero-temperature Rényi entropy of the BA model has contributions from both the exponentially dense energy levels and the true ground-state entanglement for $p \leqslant 1$. In particular, the results suggest that the true groundstate entanglement primarily determines the Rényi entropy for the $\psi$ fermions, $S_{\psi}^{(2)}$, while the large many-body density of states, arising from the large- $N$ limit, affects the Rényi entropy for $c$ fermions, $S_{c}^{(2)}$. Further exploration along this direction should prove useful in separating the two contributions. We also look into the mutual information (per site) between the two species of fermions, defined as $I(p)=\frac{1}{(1+p)} S_{c}^{(2)}+$ $\frac{p}{(1+p)} S_{c}^{(2)}-S_{c \psi}^{(2)}$. I $(p)$ has a broad peak around the critical point as shown in Fig. 6(b) (inset). Indeed, the underlying QPT has left an imprint in the entanglement entropy.

\section{E. Higher dimensional large- $N$ models: Rényi entropy in an interacting diffusive metal}

We now discuss one of the main results obtained using the path-integral formalism developed in this work. We compute the Rényi entropy of a subregion in an interacting diffusive metal. To this end, we consider the higher dimensional generalizations of the SYK-derived large- $N$ models, which were discussed in Sec. IID and can be described by a Hamiltonian with the general form given in Eq. (55). The large- $N$ equations needed to determine Rényi entropy were derived in Eq. (71) for one-dimensional large- $N$ models. Here we quantify how well our the formalism performs in predicting the Rényi entropy for these systems. As mentioned earlier [see the discussion after Eq. (71)], naturally solving the saddle-point equations for the extended system of the onedimensional chain is computationally more expensive than the zero-dimensional case since we need to retain the Green's function $G_{\sigma \sigma^{\prime}}^{(x)}\left(\tau_{1}, \tau_{2}\right)$ for each SYK dot in the chain. In particular, the time complexity for solving the equations scales linearly with the number of dots which we denote as $N_{\text {dots }}$. However, this increase in time complexity does not prevent us from accessing entanglement for large chains and we are able to perform calculations for values of $N_{\text {dots }}$ which are well beyond the reach of $\mathrm{ED}$, even for the noninteracting large- $N$ models for which we use the correlation matrix approach of Sec. II C. Therefore, we adopt the following strategy: First, we compare the results of our large- $N$ field-theoretic approach with that obtained from correlation matrix for noninteracting systems for moderate system sizes, and then explore the behavior of entanglement for large noninteracting and interacting systems. The interacting systems are much larger than that accessible via ED.

We set $q_{\text {hop }}=q=1$ in Eq. (55), making the model noninteracting, and look at two configurations of hopping amplitudes $t_{\text {chain }}=J=1$ and $t_{\text {chain }}=1, J=0$. Also, we set $N_{\text {dots }}=10$ and $N=10$, i.e., a moderate number of flavors per dot to perform exact numerics using the correlation matrix approach of Sec. IIC. This allows us to access subsystems sizes for $p=0.0-1.0$ in steps of 0.1 . The result for the above two scenarios are shown in Figs. 7(a) and 7(b) respectively. We find, like before, an excellent agreement between large- $N$ predictions and exact numerical calculations, with the results for Rényi entropy from both the techniques collapsing on top of each other for multiple temperature values.

Having established the agreement of our formalism with exact numerics, we move on to larger chain sizes involving interacting as well as noninteracting dots. In particular, we keep $q_{\text {hop }}=1$ and study chains with either $q=2$ or $q=1$ body interaction for the dots [see Eq. (55)]. The resultant ground state in these cases is known to describe either a strongly interacting diffusive Fermi liquid [32] or a noninteracting (but still diffusive) FL, both of which are gapless phases. The entanglement scaling with subsystem size $(l)$ for a FL have been argued to violate the area law. For example, in a 1-d translationally invariant gapless Fermi system, conformal field theory (CFT) predicts [2] that the von Neuman entanglement 

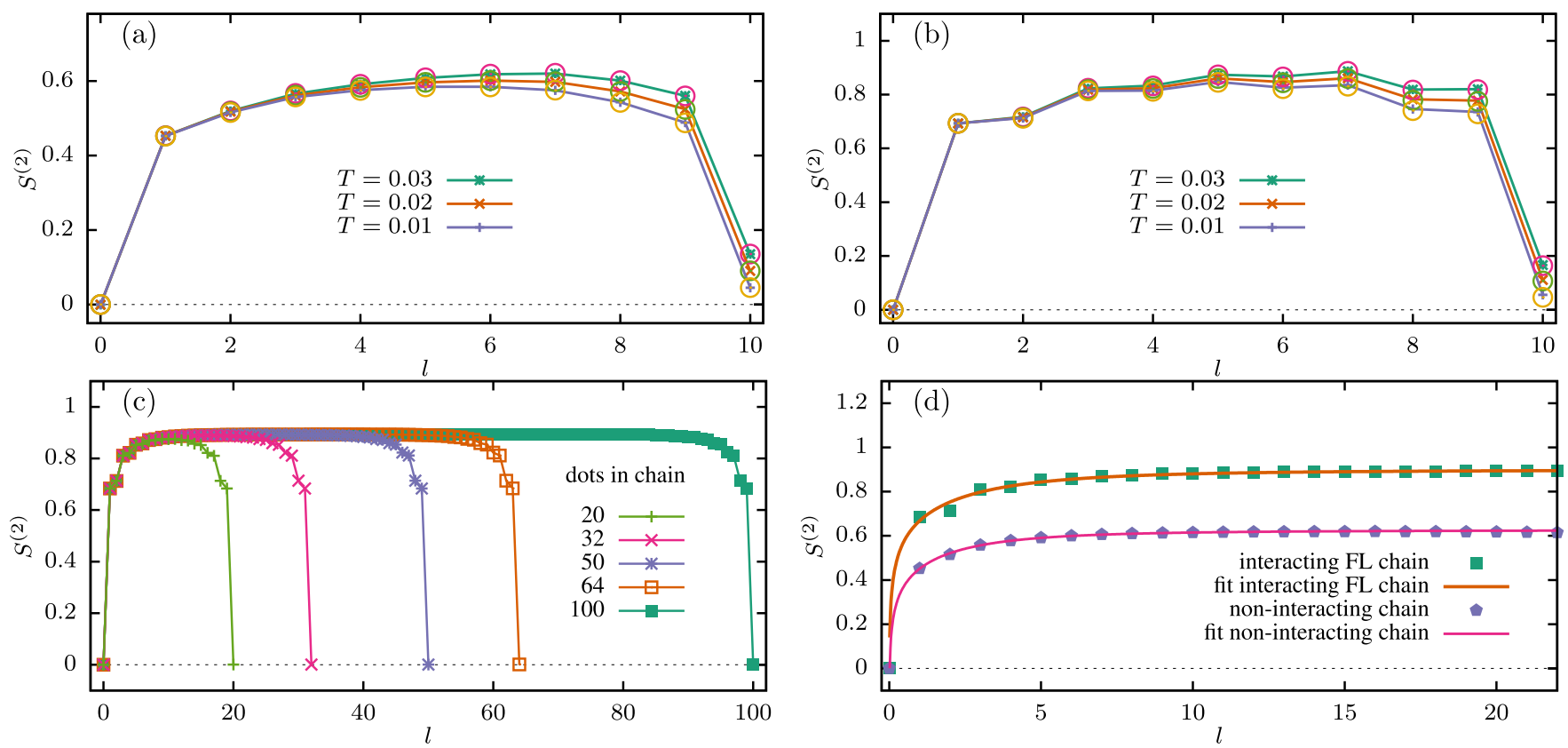

FIG. 7. Rényi entropy of diffusive Fermi liquid: (a) Behavior of Rényi entropy, $S^{(2)}$, vs subsystem size $l$ in a model of noninteracting dots coupled to nearest neighbors in a ring of $N_{\text {dots }}=10$ dots [see Fig. 2(d)], for temperatures $T=0.03,0.02,0.01$. The model is obtained by setting $q=q_{\text {hop }}=1$ and intra- as well as interdot hopping strengths $t_{\text {chain }}=J=1$ in Eq. (55). (b) Rényi entropy vs $l$ for the noninteracting model when $t_{\text {chain }}=1, J=0$. In both panels (a) and (b), large- $N$ predictions (points joined by lines) are in agreement with the results obtained from exact numerics (color matched circles) performed using correlation matrices. (c) Extrapolated zero-temperature Rényi entropy as a function of subsystem size $l$ for a ring of SYK dots [Fig. 2(d)], connected by random hoppings. We set the interaction strength $J=1$ within each SYK dot as well as the strength of hopping between dots $t_{\text {chain }}=1$. The number of dots in the chain, $N_{\text {dots }}$, is varied from 20 to 100 dots (well beyond the scope of any exact numerics), to verify that the growth of entanglement for small subsystem sizes becomes independent of $N_{\text {dots }}$ for $N_{\text {dots }}>20$. (d) An enlarged view, showing the growth of $S^{(2)}$ near small subsystem sizes, i.e., $l \leqslant 20$, in the interacting SYK chain (represented by squares) and the noninteracting chain (represented by pentagon) discussed in panel (a), for a large value of chain size $N_{\text {dots }}=50$. Both curves can be fitted (lines) with the analytical function $S^{(2)} \sim \ln \left[1 /\left(l^{-2}+l_{0}^{-2}\right)^{1 / 2}\right]$, where $l_{0}$ is an emergent "mean-free-path" length scale which takes a value between $\approx 4$ and 5 in this case.

entropy $S^{(1)}$ (obtained by taking the $\operatorname{limit}_{n \rightarrow 1} \lim _{n \rightarrow}^{(n)}$ ) scales with the universal form $S^{(1)}=(c / 3) \ln (l)+$ const., where $c$ is the central charge of the underlying CFT, following which an exact expression for $S^{(1)}$, of the form $S^{(1)} \sim l^{d-1} \ln l$, was obtained for noninteracting FLs in arbitrary dimensions $d$ [74]. Later on, it was argued that the $n$ th-order Rényi entropy for noninteracting as well as interacting FLs should follow $S^{(n)}=\frac{1}{2}\left(1+\frac{1}{n}\right) S^{(1)}$, and therefore should have the same scaling with system size [75,76]. The above scaling form has been studied and confirmed, for systems with and without interactions, using several numerical approaches [77]. Therefore, we may naively expect to recover this $\ln l$ scaling for our model of a one-dimensional chain of connected dots.

To verify this, we evaluate the secondRényi entropy, in the $T \rightarrow 0$ limit, as a function of subsystem size $l$ for increasing values of $N_{\text {dots }}$ and plot the result in Fig. 7(c). We find that the curves converge as the chain size increases and entanglement entropy growth near $l=0$ becomes independent of the total number of dots in the chain. Surprisingly, we find that Rényi entropy quickly saturates to a finite value as subsystem size is increased, a behavior inconsistent with $\ln l$ scaling, which poorly fits the the entanglement growth curve in our calculation. Instead, we find that a "modified" growth function

$$
S^{(2)}(l) \sim \ln \left[\frac{1}{\sqrt{l^{-2}+l_{0}^{-2}}}\right]+\text { const. }
$$

fits our data rather well; see Fig. 7(d). This indicates the presence of an emergent length scale $l_{0}$ in the gapless system. This could be explained by realizing that the FL state is obtained by connecting sites (dots) having random intradot couplings, which are uncorrelated at different sites, with random hoping amplitudes [e.g., $t_{i j x x^{\prime}}$ in Eq. (55)]. The former will induce an effective mean free path for the quasiparticles in the system and therefore will cut off the growth of entanglement beyond a length scale $l_{0}$. Indeed, if we identify $l_{0}$ as the mean-free path and take $l_{0} \rightarrow \infty$, we recover the expected $\ln l$ growth of entanglement. However, we note that a definition of mean free path in a system with random intersite hopping (with zero mean) is somewhat subtle due to the difficulty in identifying a Fermi velocity. Interestingly, the growth function in Eq. (88) has also been suggested in Ref. [78] based on numerical studies of system-size scaling of entanglement in a disordered noninteracting system and hydrodynamic arguments for diffusive FL. In contrast, our results explicitly demonstrate the scaling law of Eq. (88) in an interacting diffusive metal for 
system sizes much beyond any other numerical techniques. The coefficient of the growth function in Eq. (88) is also expected to contain the information of effective central charge [78] of the underlying CFT. As evident in Fig. 7(d), the coefficient of the growth function changes with interaction strength. These aspects will be studied in detail in a future work [71].

\section{CONCLUSIONS AND DISCUSSION}

In this work, we have proposed and developed an equilibrium and nonequilibrium field theory method to compute Rényi entanglement entropies for interacting fermions. The basis of the field theory formalism relies on an operator identity that we have derived here. The path-integral technique is an alternative, and maybe complementary, to the existing path-integral methods that typically require complicated boundary conditions on the fields for computing Rényi entropy. Our method rigorously transforms the complex boundary conditions into time-dependent self-energies while preserving the familiar antiperiodic boundary conditions on the fermionic fields as in a usual coherent-state path integral. As a result, the path-integral formalism could be easily incorporated within standard weak-coupling diagrammatic field-theory techniques and approximations, e.g., mean-field theory and random-phase approximation (RPA), in a very transparent manner. The path-integral technique to compute entanglement entropy could also be integrated with strong-coupling approaches, e.g., in Hubbard model, to go beyond Gaussian actions [79]. We demonstrate this by formulating the field theory for Rényi entropy in Hubbard model within DMFT approximation, and in several interacting large- $N$ fermion models of current interest.

Using the formalism, we obtain several important results on Rényi entropy of FL and NFL states in zero-dimensional interacting large- $N$ models, i.e., SYK and related models. We exactly compute the second Rényi entropy of a subsystem as a function relative subsystem size $p$ in the $N \rightarrow \infty$ limit. We analytically show that the subsystem is maximally entangled with an entanglement entropy $p \ln (2)$ at half filling in the limit $p \rightarrow 0$. By comparing with exact diagonalization result for small system sizes $N=10-12$, we demonstrate that the $1 / N$ corrections are very small for entanglement entropy for noninteracting systems as well as for strongly interacting heavy Fermi liquids. However, the $1 / N$ corrections are found to be large and nonperturbative for $p \gtrsim 0.4$ in the SYK model, where the residual entropy of the NFL state contributes to the $T \rightarrow 0$ Rényi entropy in the large- $N$ limit, as we analytically show for $p \rightarrow 1$. Thus, our results reveal intriguing connections between residual entropy of SYK NFL and its $T \rightarrow 0$ Rényi entropy in the large- $N$ limit, calling for a proper interpretation of residual Rényi entropy, in addition to the pure ground-state quantum entanglement, for a general $p$. We further make the connection between residual entropy and Rényi entropy explicit in the BA model where the difference of $T \rightarrow 0$ Rényi entropy of larger and smaller subsystems, for a particular subdivision of the system, can be directly attributed to the residual entropy of the NFL state. Finally, using our method, we obtain nontrivial system-size scaling of entanglement in an interacting diffusive metal for system sizes much beyond that accessible via exact diagonalization methods. We find the existence of an emergent length scale that limits the growth of entanglement entropy in the diffusive metal.

The DMFT formulation developed here for Rényi entropy would be very useful to understand quantum entanglement in correlated systems. The formulation could be easily integrated with a standard impurity solver, e.g., CTQMC [26]. Entanglement entropy has been previously studied within CTQMC, treating the interaction correction perturbatively for weak interaction [21]. Our method is nonperturbative and can be used in the strongly interacting regime. The trace formula and the path integral derived here might also be useful to compute Rény entropy of fermions in other QMC techniques, like determinant QMC (DQMC) [18-21,80].

The field theory developed in this work is also amenable to perturbative renormalization group (RG) methods, e.g., applied to nonequilibrium situations [81], that may be advantageous for deriving analytical results on entanglement entropy in interacting fermionic systems. It will be interesting to explore possible recursion relations between Rényi entropies at different orders $(n)$ to understand the behavior as a function of $n$ and take appropriate limit to compute von Neumann entanglement entropy and entanglement negativity [12-16] for interacting fermions. For the large- $N$ fermionic models, it will be desirable to go beyond the $N \rightarrow \infty$ limit to compute fluctuations around the saddle point of the entanglement action, e.g., by generalizing the methods of Ref. [82] for SYK thermal field theory. The time-dependent self-energy kick in the real-time action [e.g., in Eq. (D5)] can be mimicked by time-dependent non-Hermitian terms in a Hamiltonian or nonunitary terms in the time evolution. Hence, a protocol, inspired by the "kick" interpretation, may also be designed to measure the Rényi entropy in quantum circuits.

\section{ACKNOWLEDGMENTS}

We thank Rajdeep Sensarma and Saranyo Moitro for useful discussions and for collaboration at the initial stages of the work. We also thank Thomas Scaffidi for discussions with A.H. at the final stages of this work. S.B. acknowledges support from the Infosys Foundation (India) and SERB (DST, India) (ECR/2017/001742). A.H. acknowledges Compute Canada [83] for the use of their cluster framework for performing the numerical computations presented in this paper.

\section{APPENDIX A: OPERATOR EXPANSION}

In this Appendix, we derive the operator expansion identity of Eq. (3). The displacement operator defined in Sec. II A can be used to represent [25] the Dirac $\delta$ function for Grassmann numbers

$$
\delta(\xi-\eta)=\operatorname{Tr}\left[D_{N}(\xi) E_{A}(\eta)\right],
$$

where the operator $E_{A}$ is defined as

$$
E_{A}(\boldsymbol{\eta})=\int d^{2} \boldsymbol{\alpha} \exp \left[\sum_{i} \eta_{i} \bar{\alpha}_{i}-\alpha_{i} \bar{\eta}_{i}\right]|\boldsymbol{\alpha}\rangle\langle-\boldsymbol{\alpha}| .
$$

The representation of the $\delta$ function [Eq. (A1)] allows the displacement operators to form a basis for expanding an arbitrary 
operator $F$, such that

$$
F=\int d^{2} \xi \operatorname{Tr}\left[F E_{A}(\xi)\right] D_{N}(\xi)
$$

Although the operator expansion formula in Eq. (A3) is useful, it has certain drawbacks. In particular, Eq. (A3) is not amenable to a path-integral formulation with simple boundary conditions mainly because of the outer product $|\boldsymbol{\alpha}\rangle\langle-\boldsymbol{\alpha}|$ in the definition of $E_{A}$ [Eq. (A2)]. Therefore, a generalization of Eq. (A3) involving only the displacement operators $D_{N}$ is desirable. In this context, an insightful observation is to realize that even the operator $E_{A}$ can be decomposed using Eq. (A3), as shown,

$$
E_{A}(\boldsymbol{\eta})=\int d^{2} \xi \operatorname{Tr}\left[E_{A}(\boldsymbol{\eta}) E_{A}(\xi)\right] D_{N}(\xi)
$$

which can then be used to transform Eq. (A3) into

$$
F=\int d^{2} \xi, \eta f_{N}(\boldsymbol{\eta}, \boldsymbol{\xi}) \operatorname{Tr}\left[F D_{N}(\xi)\right] D_{N}(\boldsymbol{\eta})
$$

where

$$
\begin{aligned}
f_{N}(\boldsymbol{\gamma}, \boldsymbol{\xi}) \equiv & \operatorname{Tr}\left[E_{A}(\boldsymbol{\gamma}) E_{A}(\boldsymbol{\xi})\right] \\
= & 2^{N} \exp \left[\sum_{i}-\frac{1}{2}\left(\bar{\gamma}_{i} \gamma_{i}+\bar{\xi}_{i} \xi_{i}\right)\right] \\
& \times \exp \left[\sum_{i} \frac{1}{2}\left(\bar{\gamma}_{i} \xi_{i}-\bar{\xi}_{i} \gamma_{i}\right)\right] .
\end{aligned}
$$

Equation (A5) offers a way to decompose a general operator $F$ using only the normal-ordered displacement operators $D_{N}$. Using the relation between the usual displacement operators and their normal-ordered counterparts in Eq. (6), an equivalent identity involving the operator $D(\xi)$ can be derived, i.e.,

$$
F=\int d^{2} \xi, \eta f(\eta, \xi) \operatorname{Tr}[F D(\xi)] D(\eta),
$$

where

$$
\begin{aligned}
f(\boldsymbol{\eta}, \boldsymbol{\xi}) & =2^{N} \exp \left[\sum_{i} \frac{1}{2}\left(\bar{\eta}_{i} \xi_{i}-\bar{\xi}_{i} \eta_{i}\right)\right] \\
& =\exp \left[\sum_{i} \frac{1}{2}\left(\bar{\eta}_{i} \eta_{i}+\bar{\xi}_{i} \xi_{i}\right)\right] f_{N}(\boldsymbol{\eta}, \boldsymbol{\xi}) .
\end{aligned}
$$

Both the functions $f$, defined above, and $f_{N}$ [see Eq. (A6)] satisfy

$$
f_{N}(-\boldsymbol{\eta}, \boldsymbol{\xi})=f_{N}(\boldsymbol{\eta},-\boldsymbol{\xi})=f_{N}(\boldsymbol{\xi}, \boldsymbol{\eta})
$$

It can be shown that cyclic rule for trace gets modified due to the presence of the displacement operators, i.e.,

$$
\begin{aligned}
\operatorname{Tr}\left[D_{N}(\eta) G\right] & =\operatorname{Tr}\left[G D_{N}(-\eta)\right], \\
\operatorname{Tr}[D(\eta) G] & =\operatorname{Tr}[G D(-\eta)] .
\end{aligned}
$$

The trace identity of Eq. (7) can be obtained using Eqs. (3), (A9), and (A10).

\section{APPENDIX B: THE CHARACTERISTIC FUNCTION HIERARCHY}

In this, section we give the details of the steps that went into deriving the Rényi entropy hierarchy reported in Sec. II C of the main text.

\section{Derivation}

We use the operator expansion formula Eq. (A7) to write

$$
\begin{aligned}
\rho^{n+1} D(\boldsymbol{\alpha}) & =\rho^{n} \rho D(\boldsymbol{\alpha}) \\
& =\int d^{2} \boldsymbol{\xi}, \boldsymbol{\eta} f(\boldsymbol{\eta}, \boldsymbol{\xi}) \operatorname{Tr}\left[\rho^{n} D(\boldsymbol{\xi})\right] D(\boldsymbol{\eta}) \rho D(\boldsymbol{\alpha}) .
\end{aligned}
$$

Taking trace on both sides gives

$\operatorname{Tr}\left[\rho^{n+1} D(\boldsymbol{\alpha})\right]=\int d^{2} \boldsymbol{\xi}, \boldsymbol{\eta} f(\boldsymbol{\eta}, \boldsymbol{\xi}) \operatorname{Tr}\left[\rho^{n} D(\boldsymbol{\xi})\right] \operatorname{Tr}[D(\boldsymbol{\eta}) \rho D(\boldsymbol{\alpha})]$.

Using the modified cyclic rule for trace, Eq. (A11), and the product formula,

$$
D(\xi) D(\boldsymbol{\eta})=D(\xi+\eta) \exp \left[\frac{1}{2} \sum_{i}\left(\bar{\eta}_{i} \xi_{i}-\bar{\xi}_{i} \eta_{i}\right)\right],
$$

we have

$$
\begin{aligned}
\operatorname{Tr}[D(\boldsymbol{\eta}) \rho D(\boldsymbol{\alpha})] & =\operatorname{Tr}[\rho D(\boldsymbol{\alpha}) D(-\boldsymbol{\eta})] \\
& =\operatorname{Tr}[\rho D(\boldsymbol{\alpha}-\boldsymbol{\eta})] \exp \left[\frac{1}{2}\left(\sum_{i} \bar{\alpha}_{i} \eta_{i}-\bar{\eta}_{i} \alpha_{i}\right)\right] \\
& =\operatorname{Tr}[\rho D(\boldsymbol{\alpha}-\boldsymbol{\eta})] \frac{f(-\boldsymbol{\eta}, \boldsymbol{\alpha})}{2^{N_{A}}} .
\end{aligned}
$$

Putting this back, we get

$$
\begin{aligned}
\operatorname{Tr}\left[\rho^{n+1} D(\boldsymbol{\alpha})\right]= & \frac{1}{2^{N_{A}}} \int d^{2} \boldsymbol{\xi}, \boldsymbol{\eta} f(\boldsymbol{\eta}, \boldsymbol{\xi}) f(-\boldsymbol{\eta}, \boldsymbol{\alpha}) \operatorname{Tr}\left[\rho^{n} D(\boldsymbol{\xi})\right] \\
& \times \operatorname{Tr}[\rho D(\boldsymbol{\alpha}-\boldsymbol{\eta})],
\end{aligned}
$$

and substituting $\boldsymbol{\eta} \rightarrow-\boldsymbol{\eta}$, we get Eq. (38), i.e.,

$$
\begin{aligned}
\operatorname{Tr}\left[\rho^{n+1} D(\boldsymbol{\alpha})\right] \\
=\frac{1}{2^{N_{A}}} \int d^{2} \boldsymbol{\xi}, \boldsymbol{\eta} f(-\boldsymbol{\eta}, \boldsymbol{\xi}) f(\boldsymbol{\eta}, \boldsymbol{\alpha}) \\
\quad \times \operatorname{Tr}\left[\rho^{n} D(\boldsymbol{\xi})\right] \operatorname{Tr}[\rho D(\boldsymbol{\alpha}+\boldsymbol{\eta})] \\
=\frac{1}{2^{N_{A}}} \int d^{2} \boldsymbol{\xi}, \boldsymbol{\eta} f(\boldsymbol{\xi}, \boldsymbol{\eta}) f(\boldsymbol{\eta}, \boldsymbol{\alpha}) \\
\quad \times \operatorname{Tr}\left[\rho^{n} D(\boldsymbol{\xi})\right] \operatorname{Tr}[\rho D(\boldsymbol{\alpha}+\boldsymbol{\eta})],
\end{aligned}
$$

where we have used the property in Eq. (A9).

\section{Simplification of the recursion}

We continue from Eq. (38) and use the Gaussian ansatz Eq. (39) to write

$$
\begin{aligned}
\chi_{n+1}(\boldsymbol{\alpha})= & \frac{1}{2^{N_{A}}} \int d^{2} \boldsymbol{\xi}, \boldsymbol{\eta} f(\boldsymbol{\xi}, \boldsymbol{\eta}) f(\boldsymbol{\eta}, \boldsymbol{\alpha}) \chi_{n}(\boldsymbol{\xi}) \chi_{1}(\boldsymbol{\alpha}+\boldsymbol{\eta}) \\
= & \lambda_{n} \lambda \frac{1}{2^{N_{A}}} \int d^{2} \boldsymbol{\xi}, \boldsymbol{\eta} f(\boldsymbol{\xi}, \boldsymbol{\eta}) f(\boldsymbol{\eta}, \boldsymbol{\alpha}) \exp \left[\overline{\boldsymbol{\xi}}^{T} A_{n} \boldsymbol{\xi}\right] \\
& \times \exp \left[(\overline{\boldsymbol{\alpha}}+\overline{\boldsymbol{\eta}})^{\boldsymbol{T}} A(\boldsymbol{\alpha}+\boldsymbol{\eta})\right]
\end{aligned}
$$




$$
\begin{aligned}
= & 2^{N_{A}} \lambda_{n} \lambda \int d^{2} \xi, \eta \exp \left[\frac{\bar{\xi} \eta}{2}-\frac{\bar{\eta} \xi}{2}\right] \\
& \times \exp \left[\frac{\bar{\eta} \boldsymbol{\alpha}}{2}-\frac{\bar{\alpha} \eta}{2}\right] \exp \left[\bar{\xi}^{T} A_{n} \xi\right] \\
& \times \exp \left[(\overline{\boldsymbol{\alpha}}+\overline{\boldsymbol{\eta}})^{\boldsymbol{T}} A(\boldsymbol{\alpha}+\boldsymbol{\eta})\right] \\
= & 2^{N_{A}} \lambda_{n} \lambda \int d^{2} \boldsymbol{\xi}, \boldsymbol{\eta} \exp \left[\frac{\bar{\xi} \eta}{2}-\frac{\bar{\eta} \xi}{2}\right] \\
& \times \exp \left[\frac{\bar{\eta} \boldsymbol{\alpha}}{2}-\frac{\overline{\boldsymbol{\alpha}} \boldsymbol{\eta}}{2}\right] \exp \left[\overline{\boldsymbol{\xi}}^{T} A_{n} \boldsymbol{\xi}\right] \exp \left[\overline{\boldsymbol{\alpha}}^{\boldsymbol{T}} A \boldsymbol{\alpha}\right] \\
& \times \exp \left[\overline{\boldsymbol{\eta}}^{\boldsymbol{T}} A \boldsymbol{\eta}+\overline{\boldsymbol{\alpha}}^{\boldsymbol{T}} A \boldsymbol{\eta}+\overline{\boldsymbol{\eta}}^{\boldsymbol{T}} A \boldsymbol{\alpha}\right] .
\end{aligned}
$$

Integrating out $\xi$ and $\eta$, we get

$$
\begin{aligned}
\chi_{n+1}(\boldsymbol{\alpha}) \\
=2^{N_{A}} \lambda_{n} \lambda \operatorname{det}\left(-A_{n}\right) \operatorname{det}\left(-A-A_{n}^{-1} / 4\right) \\
\quad \times \exp \left[\overline{\boldsymbol{\alpha}}^{\boldsymbol{T}}\left(A-(A-\mathbf{1} / 2)\left(A+A_{n}^{-1} / 4\right)(A+\mathbf{1} / 2)\right) \boldsymbol{\alpha}\right] .
\end{aligned}
$$

Equating the answer to the Gaussian ansatz for $n+1$, we get

$$
\begin{aligned}
\lambda_{n+1} & \exp \left[\overline{\boldsymbol{\alpha}}^{\boldsymbol{T}} A_{n+1} \boldsymbol{\alpha}\right] \\
= & 2^{N_{A}} \lambda_{n} \lambda \operatorname{det}\left(-A_{n}\right) \operatorname{det}\left(-A-A_{n}^{-1} / 4\right) \\
& \times \exp \left[\overline{\boldsymbol{\alpha}}^{\boldsymbol{T}}\left(A-(A-\mathbf{1} / 2)\left(A+A_{n}^{-1} / 4\right)(A+\mathbf{1} / 2)\right) \boldsymbol{\alpha}\right],
\end{aligned}
$$

implying the following recursion relations for $\lambda_{n}$ and $\boldsymbol{A}_{n}$ :

$$
\begin{aligned}
\lambda_{n+1} & =2^{N_{A}} \lambda_{n} \lambda \operatorname{det}\left(-A_{n}\right) \operatorname{det}\left(-A-A_{n}^{-\mathbf{1}} / 4\right) \\
& =2^{N_{A}} \lambda_{n} \lambda \operatorname{det}\left(A_{n} A+\mathbf{1} / 4\right) \\
& =\operatorname{det}\left[2 A_{n} A+\mathbf{1} / 2\right] \lambda_{n}, \\
A_{n+1} & =\left(A-(A-\mathbf{1} / 2)\left(A+A_{n}^{-1} / 4\right)(A+\mathbf{1} / 2)\right) \\
& =\left(A+A_{n}\right) /\left(1+4 A A_{n}\right) .
\end{aligned}
$$

In the last line, we have chosen to simplify the expressions, by assuming that $A_{n}$ at the end will be well behaved functions of the matrix $A$, and therefore commutes with $A$, allowing us to treat all matrices as scalars. We rewrite the recursion for $A_{n}$ as

$$
\begin{aligned}
A_{n+1} A & =\left(A^{2}+A_{n} A\right) /\left(1+4 A A_{n}\right) \\
\Rightarrow 2 A_{n+1} A+\frac{1}{2} & =\left(2 A^{2}+2 A_{n} A\right) /\left(1+4 A A_{n}\right)+\frac{1}{2} \\
\Rightarrow 2 A_{n+1} A+\frac{1}{2} & =\frac{\left(2 A^{2}+2 A_{n} A\right)+\frac{1}{2}\left(1+4 A A_{n}\right)}{\left(1+4 A A_{n}\right)} \\
\Rightarrow 2 A_{n+1} A+\frac{1}{2} & =1+\frac{\left(A^{2}-\frac{1}{4}\right)}{2 A_{n} A+\frac{1}{2}} .
\end{aligned}
$$

The last line gives the expression reported in the main text as Eq. (43).

\section{Solving the recursion}

We find the following solution

$$
X_{n}=\left[\left(\mathbf{1}-C^{T}\right)^{n}+\left(C^{T}\right)^{n}\right]\left[\left(\mathbf{1}-C^{T}\right)^{n-1}+\left(C^{T}\right)^{n-1}\right]^{-1}
$$

solves Eq. (43). To prove it, we first define $B=C^{T}$ (and treat everything like scalars as explained earlier) to simplify the notation and then plug the above solution into the right-hand side of the recursion [Eq. (43)] and simplify as shown below,

$$
\begin{aligned}
1+\frac{B(B-1)}{X_{n}} & =1+\frac{B(B-1)}{\frac{(1-B)^{n}+B^{n}}{(1-B)^{n-1}+B^{n-1}}} \\
& =1+\frac{B(B-1)\left[(1-B)^{n-1}+B^{n-1}\right]}{(1-B)^{n}+B^{n}} \\
& =\frac{(1-B)^{n}+B^{n}-B(1-B)^{n}-(1-B) B^{n}}{(1-B)^{n}+B^{n}} \\
& =\frac{(1-B)^{n+1}+B^{n+1}}{(1-B)^{n}+B^{n}}=X_{n+1},
\end{aligned}
$$

to find the answer to be $X_{n+1}$. Therefore, Eq. (B10) solves the recursion in Eq. (43).

\section{APPENDIX C: KELDYSH FORMULATION FOR NONINTERACTING SYSTEMS}

For a noninteracting system undergoing nonequilibrium time evolution starting from an initial Gaussian state, e.g., described by the thermal density matrix of Eq. (28), the characteristic function [Eqs. (23) and (24)] can be obtained in the following form,

$$
\begin{aligned}
\chi_{N}[\boldsymbol{\xi}, t]= & Z^{-1} \int \mathcal{D}(\bar{c}, c) \exp \left[i \int_{\mathcal{C}} d z_{1} d z_{2}\right. \\
& \times \sum_{i j} \bar{c}_{i}\left(z_{1}\right) G_{i j}^{-1}\left(z_{1}, z_{2}\right) c_{j}\left(z_{2}\right) \\
& +\mathfrak{i} \int_{\mathcal{C}} d z \sum_{i \in A}\left[\bar{c}_{i}(z) \delta_{\mathcal{C}}\left(z,\left(t^{+},+\right)\right) \xi_{i}\right. \\
& \left.-\bar{\xi}_{i} \delta_{\mathcal{C}}(z,(t,+)) c_{i}(z)\right],
\end{aligned}
$$

where $G_{i j}\left(z_{1}, z_{2}\right)$ is the contour-ordered single-particle Green's function that encodes the details of the nonequilibrium process, e.g., for time-dependent Hamiltonian $H(t)=\sum_{i j} t_{i j}(t) c_{i}^{\dagger} c_{j} \quad$ with time-dependent hopping $t_{i j}(t), \quad G_{i j}^{-1}\left(z_{1}, z_{2}\right)=\left[\left(i \partial_{z_{1}}+\mu\right) \delta_{i j}-t_{i j}\left(t_{1}\right)\right] \delta_{\mathcal{C}}\left(z_{1}-z_{2}\right)$ $\left[z_{1}=\left(t_{1}, \pm\right)\right]$. We can integrate out the fermions to get

$$
\begin{aligned}
\chi_{N}(\xi, t) & =\exp \left[\sum_{i, j \in A} \bar{\xi}_{i}\left\{-i G_{i j}\left((t,+),\left(t^{+},+\right)\right)\right\} \xi_{j}\right] \\
& =\exp \left[\sum_{i j \in A} \bar{\xi}_{i} C_{i j}^{T}(t) \xi_{j}\right] .
\end{aligned}
$$

The last line follows from the relation $G_{i j}\left((t,+),\left(t^{+},+\right)\right)=$ $G^{\mathrm{T}}\left(t, t^{+}\right)=i\left\langle c_{j}^{\dagger}(t) c_{i}(t)\right\rangle=i C_{i j}^{T}(t)$ involving the time-ordered Green's function $G^{\mathrm{T}}$. Using Eq. (21) and the characteristic function above, the second Rényi entropy can be immediately evaluated by integrating out the auxiliary Grassmann variables $\xi$ and $\eta$ to get

$$
S_{A}^{(2)}(t)=-\operatorname{Tr} \ln \left[(\mathbf{1}-C(t))^{2}+C(t)^{2}\right],
$$

as discussed in Sec. IIC. The above is of the same form as obtained in Eq. (36) for the thermal case. This implies a similar recursion relation for higher Rényi entropies as the 
one given in Eq. (38) can be derived and solved in exactly the same way to give the final expression reported in Eq. (50) of the main text.

\section{APPENDIX D: NONEQUILIBRIUM FIELD THEORY FOR RÉNYI ENTROPY IN SYK MODEL}

The imaginary-time thermal field theoretic formulation discussed in Sec. IID for SYK model and its extensions allows us to access ground-state entanglement in these large- $N$ model. In this section, we discuss the large- $N$ SchwingerKeldysh formulation, which will enable us to track the time evolution of entanglement entropy under nonequilibrium situations [54-62]. We demonstrate this by deriving the Swinger-Keldysh action and saddle-point equations for outof-equilibrium evolution in the $\mathrm{SYK}_{q}$ model [Eq. (52)] when the strength of interactions is varying with time, such that

$$
\left\langle\left|J_{i j k l}\right|^{2}\right\rangle=J(t)^{2} / q N^{2 q-1}(q !)^{2} .
$$

Here $J(t)$ is an arbitrary function of time. Also, we take the initial density matrix as a thermal ensemble for the $\mathrm{SYK}_{q}$ model prepared for some initial configuration of $J_{i j k l} \mathrm{~s}$. We use the path integral derived in Eq. (27) and a modified form of the contour defined in Eq. (26) to develop the formulation. Instead of stretching the real-time branches to $+\infty$ [see Fig. 1(b)], we stop it at $t$, the time at which entanglement is to be measured. Therefore, the contour is now defined as

$$
\mathcal{C}=\left[t_{0}+\mathfrak{i} \beta, t_{0}\right) \cup\left[t_{0}, t\right] \cup\left(t, t_{0}\right],
$$

where the imaginary-time contour remains same as that shown in Fig. 1(b).

The steps are similar to the thermal case of Sec. II D, but with the imaginary-time $\tau$ generalized to the contour variable $z$, and we end up with an intermediate replica-diagonal Keldysh action

$$
\begin{aligned}
\mathcal{S}= & \left(-\sum_{i \in A}\left[\begin{array}{ll}
\bar{\xi}_{i} & \bar{\eta}_{i}
\end{array}\right]\left[\begin{array}{cc}
\frac{1}{2} & -\frac{1}{2} \\
\frac{1}{2} & \frac{1}{2}
\end{array}\right]\left[\begin{array}{l}
\xi_{i} \\
\eta_{i}
\end{array}\right]\right. \\
& +\int_{\mathcal{C}} d z \delta_{\mathcal{C}}\left(z_{-}-t\right)\left[\bar{c}_{i 1}(z) \quad \bar{c}_{i 2}(z)\right]\left[\begin{array}{c}
\xi_{i} \\
\eta_{i}
\end{array}\right] \\
& \left.-\int_{\mathcal{C}} d z \delta_{\mathcal{C}}(z-t)\left[\begin{array}{ll}
\xi_{i} & \bar{\eta}_{i}
\end{array}\right]\left[\begin{array}{l}
c_{i 1}(z) \\
c_{i 2}(z)
\end{array}\right]\right) \\
& +\int_{\mathcal{C}} d z_{1} \sum_{i, \sigma} \bar{c}_{i \sigma}\left(z_{1}\right) i \partial_{z_{1}} c_{i \sigma}\left(z_{1}\right) \\
& -i N \int d z_{1,2} \sum_{\sigma \sigma^{\prime}}\left[\Sigma_{\sigma \sigma^{\prime}}\left(z_{1}, z_{2}\right) G_{\sigma^{\prime} \sigma}\left(z_{2}, z_{1}\right)\right. \\
& \left.-\frac{J\left(z_{1}\right) J\left(z_{2}\right)}{2 q}\left[G_{\sigma \sigma^{\prime}}\left(z_{2}, z_{1}\right)\right]^{q}\left[G_{\sigma^{\prime} \sigma}\left(z_{1}, z_{2}\right)\right]^{q}\right],
\end{aligned}
$$

where the contour $\delta$ function $\delta_{\mathcal{C}}\left(z_{-}-t\right)$ is nonzero when $z$ approaches the measurement time $t$ along the - branch of the contour and $\delta_{\mathcal{C}}(z-t)$ is nonzero when $z$ approaches $t$ from the + branch of the contour; see Eq. (D2). The large- $N$ field $G$ is upgraded to a contour version and is defined as

$$
G_{\sigma^{\prime} \sigma}\left(z_{2}, z_{1}\right)=\frac{i}{N} \sum_{i} \bar{c}_{i \sigma}\left(z_{1}\right) c_{i \sigma^{\prime}}\left(z_{2}\right) .
$$

The rest of the symbols have the same meaning as the ones defined in Eq. (59). We emphasize that the time dependence is explicitly encoded in the function $J(z)$, which is equal to $J(t)$ when $z \in\left[t_{0}, t\right] \cup\left(t, t_{0}\right]$ part of the contour, and a static quantity $J_{0}$ when $z \in\left[t_{0}+i \beta, t_{0}\right)$. If we integrate $\xi, \eta$ first, in Eq. (D3), and then the fermion-fields $\bar{c}_{i \sigma}(z), c_{i \sigma}(z)$, in that order, we arrive at the final expression for the entanglementKeldysh action,

$$
\begin{aligned}
\mathcal{S}^{\mathcal{C}}= & -i \ln \operatorname{det}\left[-i\left(i \partial_{z}-\boldsymbol{\Sigma}\right)\right] \\
& -i p \ln \operatorname{det}\left[-i\left(i \partial_{z}-\boldsymbol{\Sigma}+i \boldsymbol{M}\right)\right] \\
& -i \int d z_{1,2} \sum_{\sigma \sigma^{\prime}}\left[\Sigma_{\sigma \sigma^{\prime}}\left(z_{1}, z_{2}\right) G_{\sigma \sigma^{\prime}}\left(z_{2}, z_{1}\right)\right. \\
& \left.-\frac{J\left(z_{1}\right) J\left(z_{2}\right)}{2 q} G\left(z_{2}, z_{1}\right)^{q} G\left(z_{1}, z_{2}\right)^{q}\right],
\end{aligned}
$$

in the large- $N$ limit. The symbols $\partial_{z}, \boldsymbol{\Sigma}$ represents matrices having elements $\partial_{z_{1}} \delta_{\mathcal{C}}\left(z_{1}-z_{2}\right) \delta_{\sigma \sigma^{\prime}}, \Sigma_{\sigma \sigma^{\prime}}\left(z_{1}, z_{2}\right)$ respectively, and the matrix $\boldsymbol{M}$ is defined as

$$
\boldsymbol{M}_{\sigma_{1} \sigma_{2}}\left(\tau_{1}, \tau_{2}\right)=\left[\begin{array}{rr}
1 & 1 \\
-1 & 1
\end{array}\right] \delta_{\mathcal{C}}\left(z_{1-}-t\right) \delta_{\mathcal{C}}\left(z_{2+}-t\right) .
$$

Equation (D5) is the time-dependent generalization of Eq. (65) that was derived in the context of thermal-field theory, and in the same manner, the saddle-point equations can be derived to yield

$$
\begin{aligned}
\boldsymbol{G} & =(1-p) \tilde{\boldsymbol{G}}+p \boldsymbol{g}, \\
\tilde{\boldsymbol{G}} & =\left(i \boldsymbol{\partial}_{z}-\mathbf{\Sigma}\right)^{-1}, \\
\boldsymbol{g} & =\left(i \boldsymbol{\partial}_{z}-\boldsymbol{\Sigma}+i \boldsymbol{M}\right)^{-1}, \\
\Sigma_{\sigma \sigma^{\prime}}\left(z_{1}, z_{2}\right) & =J\left(z_{1}\right) J\left(z_{2}\right) G_{\sigma \sigma^{\prime}}\left(z_{1}, z_{2}\right)^{q} G_{\sigma^{\prime} \sigma}\left(z_{2}, z_{1}\right)^{q-1},
\end{aligned}
$$

the solutions for which, when plugged into Eq. (D5), provide us with the expression for second Rényi entropy density at time $t$, i.e.,

$$
S^{(2)}(t)=-i \mathcal{S}^{\mathcal{C}}(t)+2 N^{-1} \ln Z,
$$

where $Z$ is the partition function describing the initial thermal ensemble and can be computed from standard thermal-field theory for the $\mathrm{SYK}_{q}$ model. The generalization of the formulation to other versions of the SYK model can be done in exactly the same manner and will be discussed elsewhere [71].

\section{APPENDIX E: SADDLE-POINT EQUATIONS AND ENTANGLEMENT FREE ENERGY FOR THE MODELS OF FL AND NFL-FL TRANSITION}

In this Appendix, we provide the saddle-point equations and entanglement free energies for the rest of the large- $N$ modules introduced in Sec. II D.

\section{SYK model with quadratic hopping term}

The saddle-point equations for the interacting Fermi liquid defined in Eq. (53) are same as Eq. (67), except the formula for the self-energy is now given by

$$
\begin{aligned}
\Sigma_{\sigma \sigma^{\prime}}\left(\tau_{1}, \tau_{2}\right)= & (-1)^{q+1} J^{2} G_{\sigma \sigma^{\prime}}\left(\tau_{1}, \tau_{2}\right)^{q} G_{\sigma^{\prime} \sigma}\left(\tau_{2}, \tau_{1}\right)^{q-1} \\
& +t_{\text {hop }}^{2} G_{\sigma \sigma^{\prime}}\left(\tau_{1}, \tau_{2}\right) .
\end{aligned}
$$


Also, the entanglement free energy becomes

$$
\begin{aligned}
F_{\mathrm{EE}}(p, \beta) & =\frac{1}{2 \beta}[p \ln \operatorname{det}(-\mathbf{g})+(1-p) \ln \operatorname{det}(-\tilde{\mathbf{G}}) \\
& -\int_{0}^{\beta} d \tau_{1,2} \sum_{\sigma=1,2}(-1)^{q} \frac{J^{2}}{2 q} G_{\sigma^{\prime} \sigma}\left(\tau_{2}, \tau_{1}\right)^{q} G_{\sigma \sigma^{\prime}}\left(\tau_{1}, \tau_{2}\right)^{q} \\
& +\int_{0}^{\beta} d \tau_{1,2} \sum_{\sigma=1,2} t_{\mathrm{hop}}^{2} G_{\sigma^{\prime} \sigma}\left(\tau_{2}, \tau_{1}\right) G_{\sigma \sigma^{\prime}}\left(\tau_{1}, \tau_{2}\right) \\
& \left.-\int_{0}^{\beta} d \tau_{1,2} \sum_{\sigma=1,2} \Sigma_{\sigma \sigma^{\prime}}\left(\tau_{1}, \tau_{2}\right) G_{\sigma^{\prime} \sigma}\left(\tau_{2}, \tau_{1}\right)\right]
\end{aligned}
$$

\section{BA model for non-Fermi liquid to Fermi liquid transition}

We determine the saddle-point equations for calculating Rényi entropy in the BA model [see Eq. (54)] for the following two subsystem choices: (a) when the subsystem is chosen to be the SYK $c$ fermions and (b) when the subsystem is formed by the noninteracting $\psi$ fermions. In both cases, $p$ represents the ratio of $\psi$-fermion sites $\left(N_{\psi}\right)$ to $c$-fermion sites $\left(N_{c}\right)$, i.e., $p=N_{\psi} / N_{c}$. The self-energies $\Sigma^{(c)}$ and $\Sigma^{(\psi)}$, for both cases (a) and (b), are given by

$$
\begin{aligned}
\Sigma_{\sigma \sigma^{\prime}}^{(c)}\left(\tau_{1}, \tau_{2}\right) & =-J^{2} G_{\sigma \sigma^{\prime}}^{(c)}\left(\tau_{1}, \tau_{2}\right)^{2} G_{\sigma^{\prime} \sigma}^{(c)}\left(\tau_{2}, \tau_{1}\right) \\
& +\sqrt{p} V^{2} G_{\sigma \sigma^{\prime}}^{(\psi)}\left(\tau_{1}, \tau_{2}\right), \\
\Sigma_{\sigma \sigma^{\prime}}^{(\psi)}\left(\tau_{1}, \tau_{2}\right) & =t_{\text {hop }}^{2} G_{\sigma \sigma^{\prime}}^{(\psi)}\left(\tau_{1}, \tau_{2}\right)+\frac{V^{2}}{\sqrt{p}} G_{\sigma \sigma^{\prime}}^{(c)}\left(\tau_{1}, \tau_{2}\right) .
\end{aligned}
$$

The equations for obtaining the Green's functions from the self-energies are

$$
\begin{aligned}
\boldsymbol{G}^{(c)} & =-\left(\boldsymbol{\partial}_{\tau}+\boldsymbol{\Sigma}^{(c)}+M\right)^{-1}, \\
\boldsymbol{G}^{(\psi)} & =-\left(\boldsymbol{\partial}_{\tau}+\boldsymbol{\Sigma}^{(\psi)}\right)^{-1},
\end{aligned}
$$

for case (a) and

$$
\begin{aligned}
\boldsymbol{G}^{(c)} & =-\left(\boldsymbol{\partial}_{\tau}+\boldsymbol{\Sigma}^{(c)}\right)^{-1}, \\
\boldsymbol{G}_{(\psi)} & =-\left(\boldsymbol{\partial}_{\tau}+\boldsymbol{\Sigma}^{(\psi)}+M\right)^{-1},
\end{aligned}
$$

for case (b). The entanglement free energy is again same for both the cases and is given by

$$
\begin{aligned}
F_{\mathrm{EE}}(p, \beta) & \\
= & \frac{1}{2 \beta(1+p)}\left[\ln \operatorname{det}\left(-\boldsymbol{G}^{(c)}\right)+p \ln \operatorname{det}\left(-\boldsymbol{G}^{(\psi)}\right)\right. \\
& -\int_{0}^{\beta} d \tau_{1,2} \sum_{\sigma \sigma^{\prime}} \frac{J^{2}}{4} G_{\sigma^{\prime} \sigma}^{(c)}\left(\tau_{2}, \tau_{1}\right)^{2} G_{\sigma \sigma^{\prime}}^{(c)}\left(\tau_{1}, \tau_{2}\right)^{2} \\
& -\int_{0}^{\beta} d \tau_{1,2} \sum_{\sigma \sigma^{\prime}} \Sigma_{\sigma \sigma^{\prime}}^{(c)}\left(\tau_{1}, \tau_{2}\right) G_{\sigma^{\prime} \sigma}^{(c)}\left(\tau_{2}, \tau_{1}\right) \\
& +p \int_{0}^{\beta} d \tau_{1,2} \sum_{\sigma \sigma^{\prime}} \frac{t_{\mathrm{hop}}^{2}}{2} G_{\sigma^{\prime} \sigma}^{(\psi)}\left(\tau_{2}, \tau_{1}\right) G_{\sigma \sigma^{\prime}}^{(\psi)}\left(\tau_{1}, \tau_{2}\right)
\end{aligned}
$$

$$
\begin{aligned}
& -p \int_{0}^{\beta} d \tau_{1,2} \sum_{\sigma \sigma^{\prime}} \Sigma_{\sigma \sigma^{\prime}}^{(\psi)}\left(\tau_{1}, \tau_{2}\right) G_{\sigma^{\prime} \sigma}^{(\psi)}\left(\tau_{2}, \tau_{1}\right) \\
& \left.+\sqrt{p} \int_{0}^{\beta} d \tau_{1,2} \sum_{\sigma \sigma^{\prime}} V^{2} G_{\sigma^{\prime} \sigma}^{(c)}\left(\tau_{2}, \tau_{1}\right) G_{\sigma \sigma^{\prime}}^{(\psi)}\left(\tau_{1}, \tau_{2}\right)\right] .
\end{aligned}
$$

\section{APPENDIX F: COUPLING CONSTANT INTEGRATION METHOD TO COMPUTE GRAND POTENTIALS}

For the $n$th Rényi entropy, we need to obtain the $n$th grand potential, which is defined as

$$
\begin{aligned}
\Omega^{(n)}(\lambda) & =-T \ln Z^{(2)}(\lambda)=-T \ln \operatorname{Tr}_{A} Z_{A}^{n}(\lambda), \\
Z_{A}(\lambda) & =\operatorname{Tr}_{B} e^{-\beta\left(H_{0}+\lambda H_{1}\right)} .
\end{aligned}
$$

Here, $H_{0}$ is a reference Hamiltonian, typically the noninteracting part, whose grand potential can be obtained easily. $H_{1}$ is the interacting part and we have multiplied by a real variable $\lambda$, which will be finally integrated. The Hamiltonian $H_{\lambda}$ is defined as $H_{\lambda}=H_{0}+\lambda H_{1}$, where $\lambda=0$ is the noninteracting limit and $\lambda=1$ is the interacting Hamiltonian of interest. $n=2$ correspond to the second Rényi potential and $n=1$ to the usual grand potential. We obtain

$$
\partial_{\lambda} \Omega^{(n)}(\lambda)=\frac{-n T}{Z^{(n)}(\lambda)} \operatorname{Tr}_{A}\left[Z_{A}^{n-1}(\lambda)\left(\operatorname{Tr}_{B} \partial_{\lambda} e^{-\beta\left(H_{0}+\lambda H_{1}\right)}\right)\right],
$$

where $\partial_{\lambda}=\partial / \partial \lambda$. It can be easily shown that [84] $\partial_{\lambda} \exp \left[-\beta\left(H_{0}+\lambda H_{1}\right)\right]=-\beta \exp \left[-\beta\left(H_{0}+\lambda H_{1}\right)\right] H_{1}, \quad$ and hence

$$
\partial_{\lambda} \Omega^{(n)}(\lambda)=\frac{n}{\lambda Z^{(n)}(\lambda)} \operatorname{Tr}_{A}\left[Z_{A}^{n-1}(\lambda)\left(\operatorname{Tr}_{B} e^{-\beta\left(H_{0}+\lambda H_{1}\right)} \lambda H_{1}\right)\right] .
$$

For $n=2$, using the trace formula [Eq. (7)] and integrating both sides over $\lambda$ from 0 to 1 we get

$$
\begin{aligned}
\Omega^{(2)}(\lambda)= & \Omega^{(2)}(0)+\int_{0}^{1} \frac{d \lambda}{\lambda} \frac{2}{Z^{(2)}(\lambda)} \int d^{2}(\boldsymbol{\xi}, \boldsymbol{\eta})\left\{f_{N}(\boldsymbol{\xi}, \boldsymbol{\eta})\right. \\
& \left.\times \operatorname{Tr}\left[e^{-\beta H_{\lambda}} D_{N}(\boldsymbol{\xi})\right] \operatorname{Tr}\left[e^{-\beta H_{\lambda}} \lambda H_{1} D_{N}(\boldsymbol{\eta})\right]\right\},
\end{aligned}
$$

where $\Omega^{(2)}(0)$ is the Rényi grand potential for the noninteracting system. As in Eq. (75a), one can construct a path-integral representation for the above using the generating function $Z^{(2)}(\lambda)$ and obtain

$$
\Omega^{(2)}=\Omega^{(2)}(0)+\int_{0}^{1} \frac{d \lambda}{\lambda}\left\langle\lambda U \sum_{i} n_{i \uparrow \alpha}(0) n_{i \downarrow \alpha}(0)\right\rangle_{Z^{(2)}(\lambda)} .
$$

Here $\alpha$ is the entanglement replica index. The important point to note here is that the expectation of $\lambda H_{1}$ with respect to $Z^{(2)}$ has to be calculated at the same time where $D_{N}(\xi)$ is inserted in the path integral, i.e., at $\tau=0$ in our case. Naively, it seems that the above requires the evaluation of a four-point function. However, one can write $\left\langle\lambda U \sum_{i} n_{i \uparrow \alpha}(0) n_{i \downarrow \alpha}(0)\right\rangle$ in terms of the single-particle Green's function. To show this, we use the 
Heisenberg equation of motion [84]

$$
\begin{aligned}
\frac{d c_{i \sigma}(\tau)}{d \tau}= & {\left[H, c_{i \sigma}(\tau)\right] } \\
= & -\sum_{j} t_{i j} c_{j \sigma}(\tau)+\mu c_{i \sigma}(\tau) \\
& -\lambda U\left(n_{i \uparrow}(\tau) c_{i \downarrow}(\tau) \delta_{\sigma \downarrow}+n_{i \downarrow}(\tau) c_{i \uparrow}(\tau) \delta_{\sigma \uparrow}\right) .
\end{aligned}
$$

From the above, we can easily show that

$$
\begin{aligned}
& \left\langle\lambda U \sum_{i} n_{i \uparrow \alpha}(0) n_{i \downarrow \alpha}(0)\right\rangle_{Z^{(2)}(\lambda)} \\
& =\frac{1}{2} \lim _{\tau^{\prime} \rightarrow \tau^{+}} \sum_{i j \sigma}\left[\left(\partial_{\tau}-\mu\right) \delta_{i j}+t_{i j}\right] G_{j \sigma, i \sigma}^{(\lambda)}\left(\tau, \tau^{\prime}\right) .
\end{aligned}
$$

Using the above in Eq. (F4), we obtain the expression for second Rényi grand potential in Eq. (85) in Sec. II E. Also, the usual thermodynamic grand potential $(n=1)$ is given by

$$
\Omega=\Omega(0)+\int_{0}^{1} \frac{d \lambda}{\lambda}\left\langle\lambda U \sum_{i} n_{i \uparrow}(\tau) n_{i \downarrow}(\tau)\right\rangle_{Z(\lambda)},
$$

and one can obtain similar expression in terms of the Green's function as shown in Eq. (86) in Sec. IIE.

\section{APPENDIX G: PROOF OF MAXIMAL ENTANGLEMENT IN SYK MODEL FOR SMALL SUBSYSTEMS}

In this section, we analytically prove that the SYK model is maximally entangled with second Rényi entropy $S_{A}^{(2)}=$ $p \ln (2)$ for $p \rightarrow 0$, i.e., when the subsystem size becomes vanishing fraction of the total system size. To this end, we start from Eq. (62), and instead of integrating out the $\xi, \eta$ variables first, we integrate the $c$ fermions and then the $\xi, \eta$ variables. This leads to $\overline{\operatorname{Tr}\left[Z_{A}^{2}\right]^{r}}=\int \mathcal{D}(\bar{c}, c, \Sigma, G) e^{-N r \mathcal{S}[\Sigma, G]}$ and an effective action under (disorder) replica symmetric and diagonal ansatz

$$
\begin{aligned}
& \mathcal{S}=-\ln \operatorname{det}\left(\partial_{\tau}+\Sigma\right)+S_{G} / r-p \ln \mathcal{K}, \\
& \mathcal{K}=2\left[\left(C_{11}-1 / 2\right)\left(C_{22}-1 / 2\right)-\left(C_{12}+1 / 2\right)\left(C_{21}-1 / 2\right)\right] .
\end{aligned}
$$

Here $r$ is the number of disorder replicas, and $S_{G}$ is obtained from Eq. (65) by plugging in the ansatz $G, \Sigma \propto \delta_{a b}$ and $C_{\alpha \gamma}=\tilde{G}_{\alpha \gamma}\left(0,0^{+}\right)$(see below for definition). The above action produces an alternate [but mathematically equivalent to Eq. (67)] set of saddle-point equations

$$
\begin{gathered}
\tilde{\boldsymbol{G}}=-\left(\boldsymbol{\partial}_{\boldsymbol{\tau}}+\boldsymbol{\Sigma}\right)^{-1}, \quad \boldsymbol{G}=\tilde{\boldsymbol{G}}-p \frac{\delta \ln \mathcal{K}}{\delta \Sigma}, \\
\Sigma_{\sigma \sigma^{\prime}}\left(\tau_{1}, \tau_{2}\right)=(-1)^{q+1} J^{2} G_{\sigma \sigma^{\prime}}\left(\tau_{1}, \tau_{2}\right)^{q} G_{\sigma^{\prime} \sigma}\left(\tau_{2}, \tau_{1}\right)^{q-1} .
\end{gathered}
$$

When $p \rightarrow 0$, the above saddle-point Green's functions are same as that of the original SYK saddle point in a thermal ensemble and $\tilde{G}_{\alpha \gamma}=G_{\alpha \gamma}=G \delta_{\alpha \gamma}$. Also, $C_{\alpha \gamma}=n \delta_{\alpha \gamma}$ with $n=\left\langle c_{i}^{\dagger}(0) c_{i}(0)\right\rangle$, the fermion density per site. Hence, using Eq. (69), the Rényi entropy density of the subsystem $A$ is simply given by

$$
S^{(2)}(p)=-p \ln \left[(1-n)^{2}+n^{2}\right] .
$$

For half filling, we get $S^{(2)}=p \ln (2)$, i.e., the maximum entanglement possible for spinless fermions.

\section{APPENDIX H: NUMERICAL TECHNIQUES}

In this section, we provide the details of the numerical techniques used to arrive at the results of Sec. III. The section contains two parts: the first part gives the details for finite- $N$ calculation and the second discusses the numerical solution of the saddle-point equations that appear in the main text.

\section{Finite- $N$ numerics}

We write the thermal-density matrix, $\rho=\exp (-\beta H) / Z$, in the eigenbasis $\left(\left\{\left|\psi_{\alpha}\right\rangle\right\}\right)$ of the Hamiltonian $H$ as follows,

$$
\rho=\sum_{\alpha} p_{\alpha}\left|\psi_{\alpha}\right\rangle\left\langle\psi_{\alpha}\right|
$$

where the probability $p_{\alpha}$ are calculated using

$$
p_{\alpha}=\exp \left(-\beta E_{\alpha}\right) / Z \text {. }
$$

The many-body energies, $E_{\alpha}$, and the eigenstates, $\left|\psi_{\alpha}\right\rangle$, are obtained by exact diagonalization (ED) of the Hamiltonian $H$. The partition function, $Z$, for a given temperature $T\left(=\beta^{-1}\right)$ is calculated from the energies $E_{\alpha}$ using

$$
Z=\sum_{\alpha} \exp \left(-\beta E_{\alpha}\right)
$$

We perform our calculations using the grand-canonical ensemble in order to include contribution from all number sectors in the Fock space for fermions. The dimension of the Hilbert space is $2^{N}$ with $N$ being the total number of fermion flavors and/or sites in the theory. The reduced density matrix $\left(\rho_{A}\right)$ for the subsystem $A$, having $N_{A}$ sites, can be calculated from the reduced density matrices of the individual states $\left|\psi_{\alpha}\right\rangle$, as follows,

$$
\rho_{A}=\operatorname{Tr}_{B} \rho=\sum_{\alpha} p_{\alpha} \operatorname{Tr}_{B}\left[\left|\psi_{\alpha}\right\rangle\left\langle\psi_{\alpha}\right|\right],
$$

where $\operatorname{Tr}_{B}$ represents the trace over the rest of the system, $B$, having $N-N_{A}$ sites. We define the reduced density matrices of the individual state $\left|\psi_{\alpha}\right\rangle$ as

$$
\rho_{A}^{(\alpha)}=\operatorname{Tr}_{B}\left[\left|\psi_{\alpha}\right\rangle\left\langle\psi_{\alpha}\right|\right],
$$

which can be calculated by rewriting $\left|\psi_{\alpha}\right\rangle$ in the form

$$
\left|\psi_{\alpha}\right\rangle=\sum_{i \in A, j \in B} \psi_{i, j}^{(\alpha)}|i\rangle_{A} \otimes|j\rangle_{B} \equiv \sum_{i \in A, j \in B} \psi_{i, j}^{(\alpha)}\left|i_{A} ; j_{B}\right\rangle,
$$

where the vectors $|i\rangle_{A}\left(i=1, \ldots, 2^{N_{A}}\right)$ span the Hilbert space for the subsystem $A$, while $|j\rangle_{B}\left(j=1, \ldots, 2^{N-N_{A}}\right)$ span that of $B$, the rest of the system. The amplitude $\psi_{i, j}^{(\alpha)}$ can be viewed as a matrix with dimension $2^{N_{A}} \times 2^{N-N_{A}}$. The tracing over the 
$B$ subsystem [see Eq. (H5)] can be performed to give

$$
\begin{aligned}
\left(\rho_{A}^{(\alpha)}\right)_{i, i^{\prime}} & =\sum_{j \in B}\left\langle i_{A} ; j_{B} \mid \psi_{\alpha}\right\rangle\left\langle\psi_{\alpha} \mid i_{A}^{\prime} ; j_{B}\right\rangle \\
& =\sum_{j \in B} \psi_{i, j}^{(\alpha)}\left(\psi_{i^{\prime}, j}^{(\alpha)}\right)^{*}
\end{aligned}
$$

where $\left(\rho_{A}^{(\alpha)}\right)_{i, i^{\prime}}$ is the $i, i^{\prime}$ th element of the reduced density matrix $\rho_{A}^{(\alpha)}$. The reduced density matrix for the subsystem $A$ can now be calculated using Eq. (H4), i.e.,

$$
\rho_{A}=\sum_{\alpha} p_{\alpha} \rho_{A}^{(\alpha)}
$$

and the second Rényi entropy, $S^{(2)}$, can finally be evaluated using

$$
S^{(2)}=-\overline{\ln \left(\operatorname{Tr}_{A}\left[\rho_{A}^{2}\right]\right)} .
$$

The $\cdots$, appearing above, denotes averaging over disorder realizations of $J_{i j k l}$ s [see Eq. (52)]. We performed averaging over 2000 disorder realizations for calculations involving $N=10$ fermionic sites and averaging over 250 realizations for system involving $N=12$ sites. The reduced density matrix $\rho_{A}$ was calculated for each new disorder realization.

\section{Solution of saddle-point equations}

We demonstrate our numerical approach by using the $\mathrm{SYK}_{q}$ model, defined in Eq. (52), as a prototype. The saddlepoint equations for the model are given in Eq. (67). We reiterate these equation here for ease of access:

$$
\begin{aligned}
\boldsymbol{G} & =(1-p) \tilde{\boldsymbol{G}}+p \boldsymbol{g}, \\
\tilde{\boldsymbol{G}} & =-\left(\boldsymbol{\partial}_{\boldsymbol{\tau}}+\boldsymbol{\Sigma}\right)^{-1}, \\
\boldsymbol{g} & =-\left(\boldsymbol{\partial}_{\boldsymbol{\tau}}+\boldsymbol{\Sigma}+\boldsymbol{M}\right)^{-1}, \\
\Sigma_{\sigma \sigma^{\prime}}\left(\tau_{1}, \tau_{2}\right) & =(-1)^{q+1} J^{2} G_{\sigma \sigma^{\prime}}\left(\tau_{1}, \tau_{2}\right)^{q} G_{\sigma^{\prime} \sigma}\left(\tau_{2}, \tau_{1}\right)^{q-1} .
\end{aligned}
$$

We discretize the domain $[0, \beta)$ for imaginary-time $\tau$ into $N_{\tau}$ segments. Since the matrix $\boldsymbol{M}$, appearing above, breaks time-translation symmetry [see Eq. (64)], we represent the Green's functions $(\boldsymbol{G}, \boldsymbol{g}$ etc.) and self-energy $(\boldsymbol{\Sigma})$ as matrices having dimensions $2 N_{\tau} \times 2 N_{\tau}$. The factor 2 accounts for the two copies of the reduced density matrix in the second Rényi entropy [see Eq. (56)], and evaluating $n$th Rényi entropy will require $n N_{\tau} \times n N_{\tau}$ matrices. The time derivative is also represented as a matrix using the finite-difference relations given in Eq. (18). The antiperiodic boundary conditions for the Green's function, i.e., $G\left(\tau_{1}+\beta, \tau_{2}\right)=-G\left(\tau_{1}, \tau_{2}\right)=$ $G\left(\tau_{1}, \tau_{2}+\beta\right)$ etc., are incorporated into the matrix representation of the derivative operator as well. With this setup, the saddle-point equations can be solved by starting from an initial guess for the Green's function $\tilde{\boldsymbol{G}}, \boldsymbol{g}$, using which the self-energy $\boldsymbol{\Sigma}$ can be determined via Eq. (H13). A new "corrected" $\tilde{\boldsymbol{G}}, \boldsymbol{g}$ can then found by performing the inverses in Eq. (H11) and Eq. (H12) numerically. The process can then be iterated until sufficient numerical convergence has been achieved.
[1] P. Calabrese and J. Cardy, Entanglement entropy and quantum field theory, J. Stat. Mech. (2004) P06002.

[2] P. Calabrese and J. Cardy, Entanglement entropy and conformal field theory, J. Phys. A: Math. Theor. 42, 504005 (2009).

[3] H. Casini and M. Huerta, Entanglement entropy in free quantum field theory, J. Phys. A: Math. Theor. 42, 504007 (2009).

[4] J. Eisert, M. Cramer, and M. B. Plenio, Colloquium: Area laws for the entanglement entropy, Rev. Mod. Phys. 82, 277 (2010).

[5] G. Refael and J. E. Moore, Criticality and entanglement in random quantum systems, J. Phys. A: Math. Theor. 42, 504010 (2009).

[6] N. Laflorencie, Quantum entanglement in condensed matter systems, Phys. Rep. 646, 1 (2016).

[7] A. Kitaev and J. Preskill, Topological Entanglement Entropy, Phys. Rev. Lett. 96, 110404 (2006).

[8] H.-C. Jiang, Z. Wang, and L. Balents, Identifying topological order by entanglement entropy, Nat. Phys. 8, 902 (2012).

[9] R. Nandkishore and D. A. Huse, Many-body localization and thermalization in quantum statistical mechanics, Annu. Rev. Condens. Matter Phys. 6, 15 (2015).

[10] E. Altman and R. Vosk, Universal dynamics and renormalization in many-body-localized systems, Annu. Rev. Condens. Matter Phys. 6, 383 (2015).

[11] D. A. Abanin, E. Altman, I. Bloch, and M. Serbyn, Colloquium: Many-body localization, thermalization, and entanglement, Rev. Mod. Phys. 91, 021001 (2019).
[12] G. Vidal and R. F. Werner, Computable measure of entanglement, Phys. Rev. A 65, 032314 (2002).

[13] P. Calabrese, J. Cardy, and E. Tonni, Entanglement Negativity in Quantum Field Theory, Phys. Rev. Lett. 109, 130502 (2012).

[14] Y. A. Lee and G. Vidal, Entanglement negativity and topological order, Phys. Rev. A 88, 042318 (2013).

[15] K.-H. Wu, T.-C. Lu, C.-M. Chung, Y.-J. Kao, and T. Grover, Entanglement Renyi negativity across a finite temperature transition: A Monte Carlo study, arXiv:1912.03313 [condmat.str-el].

[16] T.-C. Lu, T. H. Hsieh, and T. Grover, Detecting topological order at finite temperature using entanglement negativity, Phys. Rev. Lett. 125, 116801 (2020).

[17] G. Vidal, J. I. Latorre, E. Rico, and A. Kitaev, Entanglement in Quantum Critical Phenomena, Phys. Rev. Lett. 90, 227902 (2003).

[18] M. B. Hastings, I. González, A. B. Kallin, and R. G. Melko, Measuring Renyi Entanglement Entropy in Quantum Monte Carlo Simulations, Phys. Rev. Lett. 104, 157201 (2010).

[19] S. Humeniuk and T. Roscilde, Quantum Monte Carlo calculation of entanglement Rényi entropies for generic quantum systems, Phys. Rev. B 86, 235116 (2012).

[20] T. Grover, Entanglement of Interacting Fermions in Quantum Monte Carlo Calculations, Phys. Rev. Lett. 111, 130402 (2013).

[21] L. Wang and M. Troyer, Renyi Entanglement Entropy of Interacting Fermions Calculated Using the Continuous-Time 
Quantum Monte Carlo Method, Phys. Rev. Lett. 113, 110401 (2014).

[22] K. E. Cahill and R. J. Glauber, Density operators and quasiprobability distributions, Phys. Rev. 177, 1882 (1969).

[23] A. Chakraborty and R. Sensarma, Wigner function and entanglement entropy for bosons from non-equilibrium field theory, arXiv:1810.10545 [cond-mat.stat-mech].

[24] S. Moitra and R. Sensarma, Entanglement entropy of fermions from Wigner functions: Excited states and open quantum systems, arXiv:2006.16271 [cond-mat.stat-mech].

[25] K. E. Cahill and R. J. Glauber, Density operators for fermions, Phys. Rev. A 59, 1538 (1999).

[26] E. Gull, A. J. Millis, A. I. Lichtenstein, A. N. Rubtsov, M. Troyer, and P. Werner, Continuous-time Monte Carlo methods for quantum impurity models, Rev. Mod. Phys. 83, 349 (2011).

[27] A. Kitaev, A simple model of quantum holography [http: //online.kitp.ucsb.edu/online/entangled15/kitaev/ and online. kitp.ucsb.edu/online/entangled15/kitaev2/].

[28] S. Sachdev and J. Ye, Gapless Spin-Fluid Ground State in a Random Quantum Heisenberg Magnet, Phys. Rev. Lett. 70, 3339 (1993).

[29] A. Georges, G. Kotliar, W. Krauth, and M. J. Rozenberg, Dynamical mean-field theory of strongly correlated fermion systems and the limit of infinite dimensions, Rev. Mod. Phys. 68, 13 (1996).

[30] S. Banerjee and E. Altman, Solvable model for a dynamical quantum phase transition from fast to slow scrambling, Phys. Rev. B 95, 134302 (2017).

[31] Y. Gu, X.-L. Qi, and D. Stanford, Local criticality, diffusion, and chaos in generalized Sachdev-Ye-Kitaev models, J. High Energy Phys. 05 (2017) 125.

[32] X.-Y. Song, C.-M. Jian, and L. Balents, Strongly Correlated Metal Built from Sachdev-Ye-Kitaev Models, Phys. Rev. Lett. 119, 216601 (2017).

[33] S. Sachdev, Bekenstein-Hawking Entropy and Strange Metals, Phys. Rev. X 5, 041025 (2015).

[34] A. Kitaev and S. J. Suh, Statistical mechanics of a twodimensional black hole, J. High Energ. Phys. 05 (2019) 198.

[35] J. Maldacena and D. Stanford, Remarks on the Sachdev-YeKitaev model, Phys. Rev. D 94, 106002 (2016).

[36] A. Altland and B. D. Simons, Condensed Matter Field Theory, 2nd ed. (Cambridge University Press, Cambridge, UK, 2010).

[37] A. Kamenev, Field Theory of Non-equilibrium Systems, 1st ed. (Cambridge University Press, Cambridge, UK, 2011).

[38] G. Stefanucci and R. van Leeuwen, Nonequilibrium Many-Body Theory of Quantum Systems: A Modern Introduction, 1st ed. (Cambridge University Press, Cambridge, UK, 2013).

[39] M. Moeckel and S. Kehrein, Real-time evolution for weak interaction quenches in quantum systems, Ann. Phys. 324, 2146 (2009).

[40] J. Dziarmaga, Dynamics of a quantum phase transition and relaxation to a steady state, Adv. Phys. 59, 1063 (2010).

[41] A. Polkovnikov, K. Sengupta, A. Silva, and M. Vengalattore, Colloquium: Nonequilibrium dynamics of closed interacting quantum systems, Rev. Mod. Phys. 83, 863 (2011).

[42] F. H. L. Essler and M. Fagotti, Quench dynamics and relaxation in isolated integrable quantum spin chains, J. Stat. Mech. (2016) 064002.

[43] Y. Gu, A. Lucas, and X.-L. Qi, Spread of entanglement in a Sachdev-Ye-Kitaev chain, J. High Energy Phys. 09 (2017) 120.
[44] S.-K. Jian and H. Yao, Solvable Sachdev-Ye-Kitaev Models in Higher Dimensions: From Diffusion to Many-Body Localization, Phys. Rev. Lett. 119, 206602 (2017).

[45] R. A. Davison, W. Fu, A. Georges, Y. Gu, K. Jensen, and S. Sachdev, Thermoelectric transport in disordered metals without quasiparticles: The Sachdev-Ye-Kitaev models and holography, Phys. Rev. B 95, 155131 (2017).

[46] A. A. Patel, J. McGreevy, D. P. Arovas, and S. Sachdev, Magnetotransport in a Model of a Disordered Strange Metal, Phys. Rev. X 8, 021049 (2018).

[47] D. Chowdhury, Y. Werman, E. Berg, and T. Senthil, Translationally Invariant Non-Fermi-Liquid Metals with Critical Fermi Surfaces: Solvable Models, Phys. Rev. X 8, 031024 (2018).

[48] A. Haldar, S. Banerjee, and V. B. Shenoy, Higher-dimensional Sachdev-Ye-Kitaev non-Fermi liquids at Lifshitz transitions, Phys. Rev. B 97, 241106(R) (2018).

[49] A. Haldar and V. B. Shenoy, Strange half-metals and Mott insulators in Sachdev-Ye-Kitaev models, Phys. Rev. B 98, 165135 (2018).

[50] D. Hauck, M. J. Klug, I. Esterlis, and J. Schmalian, Eliashberg equations for an electron-phonon version of the Sachdev-YeKitaev model: Pair breaking in non-Fermi liquid superconductors, Ann. Phys. 417, 168120 (2020).

[51] D. Chowdhury and E. Berg, Intrinsic superconducting instabilities of a solvable model for an incoherent metal, Phys. Rev. Res. 2, 013301 (2020).

[52] C.-M. Jian, Z. Bi, and C. Xu, A model for continuous thermal metal to insulator transition, Phys. Rev. B 96, 115122 (2017).

[53] O. Can and M. Franz, Solvable model for quantum criticality between the Sachdev-Ye-Kitaev liquid and a disordered Fermi liquid, Phys. Rev. B 100, 045124 (2019).

[54] A. Eberlein, V. Kasper, S. Sachdev, and J. Steinberg, Quantum quench of the Sachdev-Ye-Kitaev model, Phys. Rev. B 96, 205123 (2017).

[55] J. Sonner and M. Vielma, Eigenstate thermalization in the Sachdev-Ye-Kitaev model, J. High Energy Phys. 11 (2017) 149.

[56] I. Kourkoulou and J. Maldacena, Pure states in the SYK model and nearly $\mathrm{AdS}_{2}$ gravity, arXiv:1707.02325 [hep-th].

[57] R. Bhattacharya, D. P. Jatkar, and N. Sorokhaibam, Quantum quenches and thermalization in SYK models, J. High Energ. Phys. 07 (2019) 066.

[58] A. Haldar, P. Haldar, S. Bera, I. Mandal, and S. Banerjee, Quench, thermalization, and residual entropy across a nonFermi liquid to Fermi liquid transition, Phys. Rev. Res. 2, 013307 (2020).

[59] P. Zhang, Evaporation dynamics of the Sachdev-Ye-Kitaev model, Phys. Rev. B 100, 245104 (2019).

[60] A. Almheiri, A. Milekhin, and B. Swingle, Universal constraints on energy flow and SYK thermalization, arXiv:1912.04912 [hep-th].

[61] J. Maldacena and A. Milekhin, SYK wormhole formation in real time, arXiv:1912.03276 [hep-th].

[62] C. Kuhlenkamp and M. Knap, Periodically Driven Sachdev-YeKitaev Models, Phys. Rev. Lett. 124, 106401 (2020).

[63] C. Liu, X. Chen, and L. Balents, Quantum entanglement of the Sachdev-Ye-Kitaev models, Phys. Rev. B 97, 245126 (2018).

[64] Y. Huang and Y. Gu, Eigenstate entanglement in the SachdevYe-Kitaev model, Phys. Rev. D 100, 041901 (2019). 
[65] Y. Chen and P. Zhang, Entanglement entropy of two coupled SYK models and eternal traversable wormhole, J. High Energy Phys. 07 (2019) 033.

[66] P. Zhang, C. Liu, and X. Chen, Subsystem Rényi entropy of thermal ensembles for SYK-like models, SciPost Phys. 8, 094 (2020).

[67] P. Zhang, Entanglement entropy and its quench dynamics for pure states of the Sachdev-Ye-Kitaev model, J. High Energy Phys. 06 (2020) 143.

[68] T. A. Maier, M. Jarrell, T. Pruschke, and M. Hettler, Quantum cluster theories, Rev. Mod. Phys. 77, 1027 (2005).

[69] S. Banerjee and A. Haldar (unpublished).

[70] H. Aoki, N. Tsuji, M. Eckstein, M. Kollar, T. Oka, and P. Werner, Nonequilibrium dynamical mean-field theory and its applications, Rev. Mod. Phys. 86, 779 (2014).

[71] A. Haldar, S. Bera, and S. Banerjee (unpublished).

[72] W. Fu and S. Sachdev, Numerical study of fermion and boson models with infinite-range random interactions, Phys. Rev. B 94, 035135 (2016).

[73] W. Fu, The Sachdev-Ye-Kitaev model and matter without quasiparticles, Ph.D. thesis, Harvard University, Cambridge, MA, 2018 .

[74] D. Gioev and I. Klich, Entanglement Entropy of Fermions in Any Dimension and the Widom Conjecture, Phys. Rev. Lett. 96, 100503 (2006).
[75] B. Swingle, Entanglement Entropy and the Fermi Surface, Phys. Rev. Lett. 105, 050502 (2010).

[76] B. Swingle, Rényi entropy, mutual information, and fluctuation properties of Fermi liquids, Phys. Rev. B 86, 045109 (2012).

[77] J. McMinis and N. M. Tubman, Renyi entropy of the interacting Fermi liquid, Phys. Rev. B 87, 081108(R) (2013).

[78] A. C Potter, Boundary-law scaling of entanglement entropy in diffusive metals, arXiv:1408.1094.

[79] R. Ghosh, N. Dupuis, A. Sen, and K. Sengupta, Entanglement measures and non-equilibrium dynamics of quantum manybody systems: A path integral approach, Phys. Rev. B 101, 245130 (2020).

[80] F. F. Assaad and H. G. Evertz, World-line and determinantal quantum Monte Carlo methods for spins, phonons and electrons, in Computational Many-Particle Physics, edited by $\mathrm{H}$. Fehske, R. Schneider, and A. Weiße (Springer, Berlin, 2008), pp. 277-356.

[81] S. D. Sarkar, R. Sensarma, and K. Sengupta, A perturbative renormalization group approach to driven quantum systems, J. Phys.: Condens. Matter 26, 325602 (2014).

[82] D. Bagrets, A. Altland, and A. Kamenev, Sachdev-Ye-Kitaev model as Liouville quantum mechanics, Nucl. Phys. B 911, 191 (2016).

[83] www.computecanada.ca.

[84] A. Fetter and J. Walecka, Quantum Theory of Many-Particle Systems (McGraw-Hill, New York, 1971). 